\title{
Unconventional Continuous Structural Disorder at the Order-Disorder Phase Transition in the Hexagonal Manganites
}

\author{
Sandra H. Skjærv $\varnothing,{ }^{1, *}$ Quintin N. Meier, ${ }^{2, *}$ Mikhail Feygenson, ${ }^{3,4}$ Nicola A. Spaldin, ${ }^{2}$ \\ Simon J. L. Billinge, ${ }^{5,6}$ Emil S. Bozin, ${ }^{5, \$}$ and Sverre M. Selbach ${ }^{1, \dagger}$ \\ ${ }^{1}$ NTNU Norwegian University of Science and Technology, Department of Materials Science and \\ Engineering, NO-7491 Trondheim, Norway \\ ${ }^{2}$ ETH Zürich, Materials Theory, Wolfgang Pauli Strasse 27, CH-8093 Zürich, Switzerland \\ ${ }^{3}$ Forschungszentrum Jülich, JCNS, D-52425 Jülich, Germany \\ ${ }^{4}$ Chemical and Engineering Materials Division, Oak Ridge National Laboratory, \\ Oak Ridge, Tennessee 37831, USA \\ ${ }^{5}$ Brookhaven National Laboratory, Condensed Matter Physics and Materials Science Department, \\ Upton, New York 11973, USA \\ ${ }^{6}$ Columbia University, Department of Applied Physics and Applied Mathematics, \\ New York, New York 10027, USA
}

(Received 13 April 2018; revised manuscript received 18 March 2019; published 1 July 2019)

\begin{abstract}
The improper ferroelectricity in $\mathrm{YMnO}_{3}$ and other related multiferroic hexagonal manganites is known to cause topologically protected ferroelectric domains that give rise to rich and diverse physical phenomena. The local structure and structural coherence across the ferroelectric transition, however, were previously not well understood. Here, we reveal the evolution of the local structure with temperature in $\mathrm{YMnO}_{3}$ using neutron total scattering techniques, and we interpret them with the help of first-principles calculations and with a first-principles-based effective Hamiltonian. The results show that, at room temperature, the local and average structures are consistent with the established ferroelectric $P 6_{3} \mathrm{~cm}$ symmetry. On heating, both local and average structural analyses show striking anomalies from about $800 \mathrm{~K}$ up to the Curie temperature and signatures of a locally more preserved structure than on average, consistent with increasing fluctuations of the order-parameter angle. These fluctuations result in an unusual local symmetry lowering into a continuum of structures on heating. This local symmetry breaking persists into the high-symmetry nonpolar phase, constituting an unconventional type of order-disorder transition, and we pinpoint it as the reason for the anomalous behavior near the phase transition. The hidden disorder revealed in $\mathrm{YMnO}_{3}$ by total scattering is expected to find analogies in other materials with structural frustration or characteristic energy barriers of different magnitudes.
\end{abstract}

DOI: 10.1103/PhysRevX.9.031001

\section{INTRODUCTION}

The multiferroic hexagonal manganites, $\mathrm{h}-\mathrm{RMnO}_{3}$ $(R=\mathrm{Dy}-\mathrm{Lu}, \mathrm{In}, \mathrm{Y}$, or $\mathrm{Sc})$, are improper ferroelectrics where the polarization emerges as a secondary effect due to an improper coupling to the primary distortion mode. This results in unusual ferroelectric domain

\footnotetext{
*These co-authors contributed equally to this work.

${ }^{\dagger}$ Corresponding author.

selbach@ntnu.no

Corresponding author.

bozin@bnl.gov
}

Published by the American Physical Society under the terms of the Creative Commons Attribution 4.0 International license. Further distribution of this work must maintain attribution to the author(s) and the published article's title, journal citation, and DOI.

\section{Subject Areas: Condensed Matter Physics,}

Materials Science structures in which topological protection of the domainwall intersections causes fundamentally and technologically interesting physical properties ranging from early universe analogues [1-3] to nanoscale conducting channels [4-11]. In spite of multiple studies, the evolution of the polarization with temperature has not been explained on a microscopic level. In particular, studies based on powder neutron [12,13] and x-ray [13-18] diffraction show good agreement with a polar model describing the average structure at low and intermediate temperatures, while structural anomalies have been reported between $800 \mathrm{~K}$ and the Curie temperature $T_{\mathrm{C}}$. These findings led to a range of reported values for $T_{\mathrm{C}}$ and proposals of two distinct structural phase transitions [12-16,19], although it is now understood that there is in fact only one phase transition at $T_{\mathrm{C}}$, with the polarization slowly emerging as a secondary effect [20-24]. 
(a) Nonpolar

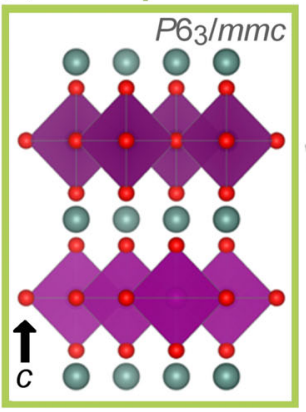

(b)

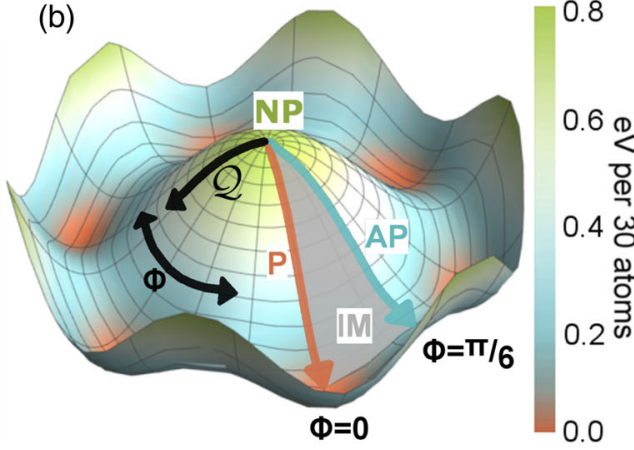

(c)

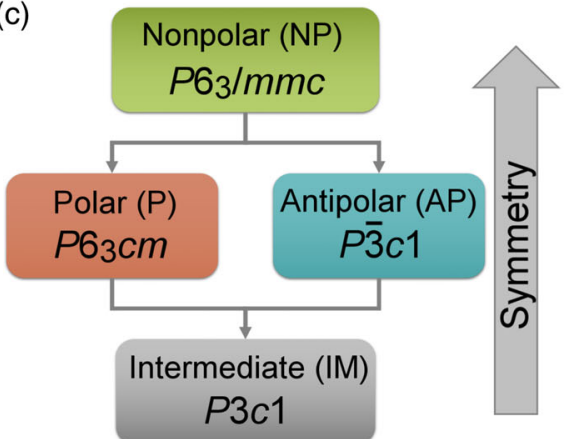

(d)

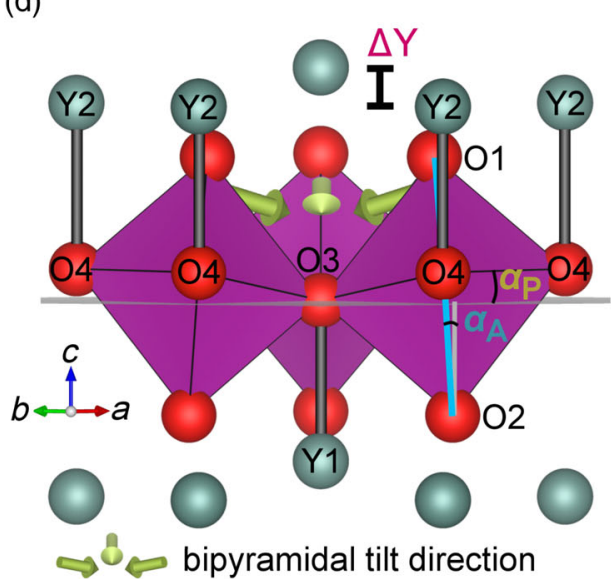

(e) Polar

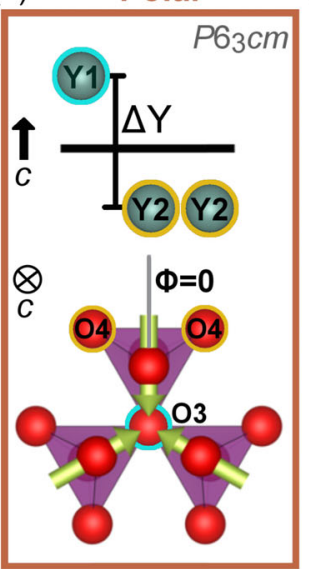

(f) Intermediate

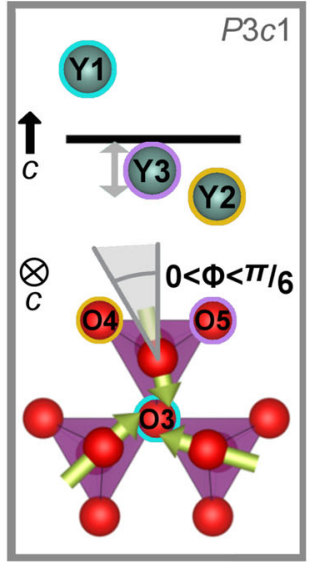

(g) Antipolar

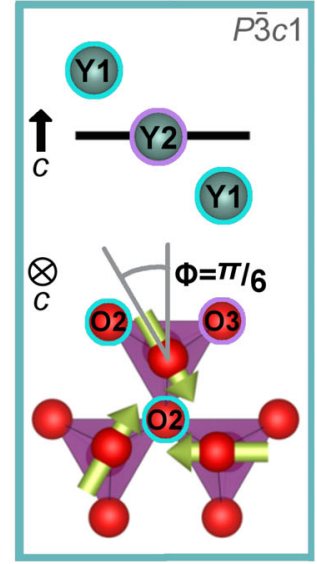

FIG. 1. Structures of $\mathrm{YMnO}_{3}$ phases. (a) The high-symmetry nonpolar (NP) structure with purple $\mathrm{MnO}_{5}$ bipyramids and turquoise Y cations. Oxygens are shown in red. (b) The Landau free energy of the hexagonal manganites as a function of the two-component order parameter $(\mathcal{Q}, \Phi)$ resembling a Mexican hat, with the NP structure at the top. In the brim of the hat, the polar (P), antipolar (AP), and intermediate (IM) subgroup structures occur at the minima, maxima, and intermediate regions, respectively. (c) Group-subgroup relationship between the high-symmetry nonpolar structure and the subgroup structures found in the brim of the Mexican-hat energy landscape. (d) The order-parameter observables for the polar subgroup are the bipyramidal tilt amplitude (angles $\alpha_{\mathrm{A}}$ and $\alpha_{\mathrm{P}}$ ) and corrugation of $\mathrm{Y}$ cations $(\Delta \mathrm{Y})$. Note that $\Delta \mathrm{Y}$ denotes the distance in the $c$ direction between $\mathrm{Y} 1$ and $\mathrm{Y} 2$. Green arrows indicate the directions of the bipyramidal tilts. The angle between the O1-O2 line and the $c$ axis defines the apical tilt $\alpha_{\mathrm{A}}$ and is a robust measure of the order-parameter amplitude $\mathcal{Q}$ irrespective of the value of $\Phi$. The plane through the three in-plane oxygens (one O3 and two O4) relative to the $a b$ plane defines the planar tilt angle $\alpha_{P}$ and is related to both the order-parameter amplitude $\mathcal{Q}$ and angle $\Phi$. (e)-(g), Structures of the subgroups at different order-parameter angles $\Phi$ : Y off-centering pattern (top) and bipyramidal tilting directions, indicated by green arrows (bottom). Atomic sites for the three subgroups are labeled and coded with colored circles to emphasize which positions are symmetry related in each phase. Atom positions with the same color markings are aligned along the $c$ axis and have the same multiplicity. A detailed overview of the atomic positions for the space groups is given in Fig. S1 of the Supplemental Material [26].

The high-symmetry nonpolar average structure of the prototypical hexagonal manganite $\mathrm{h}-\mathrm{YMnO}_{3}$ above $T_{\mathrm{C}}$ displays $P 6_{3} / m m c$ symmetry. This structure features corner-sharing $\mathrm{MnO}_{5}$ trigonal bipyramids separated by layers of $\mathrm{Y}^{3+}$ ions, each coordinated by eight oxygens [Fig. 1(a)]. The unit cell triples across $T_{\mathrm{C}}$ at around $1250 \mathrm{~K}$, as a noncentrosymmetric, but zero-polarization, zone-boundary $K_{3}$ mode condenses [25]. This $K_{3}$ distortion consists of $z$-axis displacements of the $\mathrm{Y}$ atoms and a corresponding tilt of the trigonal bipyramids. It is described by a two-component order parameter $(\mathcal{Q}, \Phi)$ with amplitude $\mathcal{Q}$ and angle $\Phi$ [21]. Here, $\mathcal{Q}$ is related to the magnitude of bipyramidal tilting and $\mathrm{Y}$ displacements, and $\Phi$ is related to the direction of the bipyramidal tilting and the displacement pattern of the Y, e.g., updown-down.

The resulting energy landscape resembles a Mexican hat [Fig. 1(b)] with three lower-symmetry space groups in the brim. The group-subgroup relationships are illustrated in Fig. 1(c). The subgroups are characterized by the angle of the order parameter $\Phi$. A general angle leads to $P 3 c 1$ symmetry [IM, Fig. 1(e)], but special values of $\Phi$ lead to higher symmetries. For $\Phi=n(\pi / 3)$, with $n=0,1, \ldots, 5$, the system is polar with space group symmetry $P 6_{3} \mathrm{~cm}$ [P, Figs. 1(d) and 1(e)]. For $\Phi=(\pi / 6)(2 n+1)$, the 
system becomes antipolar with space group symmetry $P \overline{3} c 1$ [AP, Fig. $1(\mathrm{~g})]$.

Landau theory analysis of the $K_{3}$ mode following Artyukhin et al. [21], in combination with first-principles calculations of hexagonal $\mathrm{YMnO}_{3}$, suggests an insignificantly small energy difference between the polar and antipolar symmetry when only the $K_{3}$ mode is included. Stabilization of the polar state occurs only when the $K_{3}$ mode couples to a polar $\Gamma_{2}^{-}$mode that causes a shift of the $\mathrm{Y}$ atoms towards the $\mathrm{Mn}-\mathrm{O}$ layer. Because of this improper coupling, nearly all $\Phi$ angles result in a net polarization that reaches its maximum in the $\mathrm{P}$ structure (around $6 \mu \mathrm{Ccm}^{-2}$ at room temperature) but vanishes for the AP structure. The $\mathrm{P}$ structure becomes favored by about $100 \mathrm{meV}$ per unit cell in comparison with the AP structure and by more than $600 \mathrm{meV}$ per unit cell in comparison with the NP structure at $0 \mathrm{~K}$ [21]. The exact values of the relative energies are highly temperature dependent. The resulting $Z_{6}$ symmetry of the configuration space causes the unusual sixfold ferroelectric domain patterns characteristic of h- $\mathrm{YMnO}_{3}$ [27].

This established model [21] of the Mexican-hat Landau free energy [Fig. 1(b)] describes the average symmetry evolution of the system reduced to the degrees of freedom given by the order parameter, but it does not address the underlying microscopics. In particular, whether the transition mechanism is closer to the displacive limit [the order parameter $(\mathcal{Q}, \Phi)$ goes to zero both locally and on average at $T_{\mathrm{C}}$ ] or the order-disorder limit (local order parameter is conserved) is not known.

Here, we provide such a local-structure description of the atomic structure of $\mathrm{h}-\mathrm{YMnO}_{3}$ from ambient temperature to $1273 \mathrm{~K}$, across the ferroelectric transition $T_{\mathrm{C}}$. We combine pair distribution function (PDF) analysis of neutron total scattering data with conventional Rietveld refinement to probe structural coherence and to distinguish short-range from average long-range order. This analysis reveals a surprising and unconventional behavior of the local structure as a function of temperature that cannot be explained either by a conventional order-disorder or by a displacive transition picture. In a conventional order-disorder transition, the low-temperature distortions persist above the transition in the local structure but are not evident in the long-range ordered structure due to averaging over variants of the distorted structure [28-30]. Our PDF results show that below about $800 \mathrm{~K}$, the average and local structures evolve consistently, with smoothly decreasing distortions related to a decreased order-parameter amplitude. However, between about $800 \mathrm{~K}$ and $T_{\mathrm{C}}$, the average and local structures progressively diverge from each other. Upon crossing $T_{\mathrm{C}}$, the local structure below 16-20 $\AA$ changes significantly less than the average structure, suggesting an order-disorder transition. The fit of the polar model to the local structure gradually deteriorates, while fits with the antipolar structure gradually improve, consistent with increasing fluctuations of the order-parameter angle $\Phi$ on heating. At $T_{\mathrm{C}}$, where long-range cell tripling disappears in the average structure, the fits of the polar and antipolar models become equivalent, though neither model fits ideally. The transition at $T_{\mathrm{C}}$ is therefore an order-disorder transition in the sense that the average structure transitions to the undistorted high-symmetry nonpolar structure, while the structural distortions persist in the local structure. In other words, the amplitude $\mathcal{Q}$ of the order parameter never goes to zero in the local structure. However, it is unconventional in the sense that the disordering does not occur only between the local polar variants but between all possible angles of the order parameter. These total scattering results are corroborated by a first-principles-based effective Hamiltonian predicting the same behavior.

These two key discoveries-the unconventional nature of the order-disorder transition and the extensive temperature region of symmetry-lowering fluctuations below $T_{\mathrm{C}}$ where the material accesses a wide range of structures, intermediate between the polar and antipolar subgroups, at the local scale-reconcile previous inconsistencies in the literature. Such hidden disorder, revealed by total scattering, is also anticipated in other materials where competing interactions give rise to structural frustration, or where energy barriers of different magnitudes give rise to different thermal evolution of order-parameter components.

\section{STRUCTURAL DESCRIPTION OF THE ORDER PARAMETER}

The order parameter $(\mathcal{Q}, \Phi)$ is related to three observable atomic displacements [Fig. 1(d)]: The first, $\alpha_{\mathrm{A}}$, is the bipyramidal tilt angle calculated from the line connecting the apical oxygens $(\mathrm{O} 1, \mathrm{O} 2)$ relative to the $c$ axis; the second, $\alpha_{\mathrm{P}}$, is the bipyramidal tilt of the plane through the three planar oxygens $(\mathrm{O} 3, \mathrm{O} 4)$ relative to the $a b$ plane; and the third is the out-of-plane off-centering (corrugation) of the yttrium ions, $\Delta \mathrm{Y}=c\left(z_{\mathrm{Y} 1}-z_{\mathrm{Y} 2}\right)$. The latter two are strictly defined only for the polar ground-state symmetry [Fig. 1(d)]. Shifting the order-parameter angle $\Phi$ away from the polar symmetry and towards the antipolar or intermediate space groups breaks the symmetry of the $\mathrm{Y} 2$ and $\mathrm{O} 4$ sites such that the corresponding order-parameter observables $\alpha_{\mathrm{P}}$ and $\Delta \mathrm{Y}$ have to be calculated by including the additional Wyckoff sites (see Fig. S1 in the Supplemental Material [26]).

\section{RESULTS}

\section{A. Average structure}

We first consider the average structure behavior based on qualitative and quantitative assessment of our neutron powder diffraction data. On the qualitative side, our reciprocal space data show that the (102) and (202) super-reflections of the polar structure disappear above $1223 \mathrm{~K}$, corresponding to the ferroelectric Curie temperature $T_{\mathrm{C}}$ (see Fig. S2 in the Supplemental Material [26]), in line with previous reports $[12,15,18,24]$. 


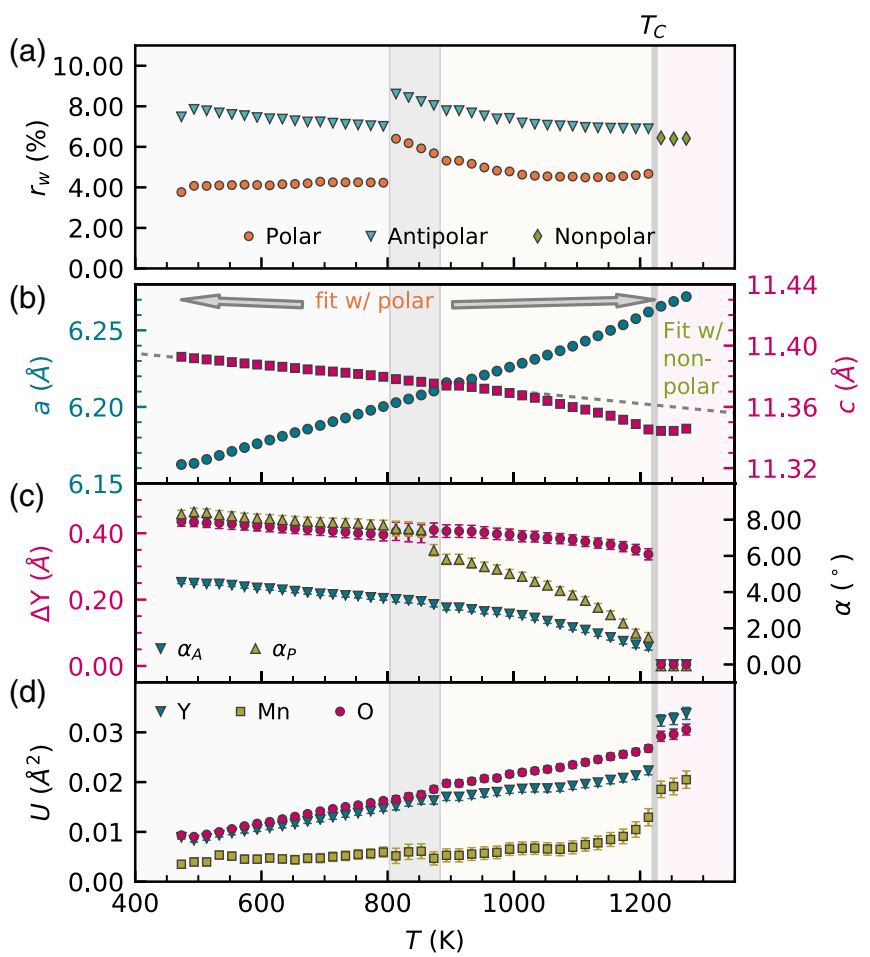

FIG. 2. Average structure refinements of $\mathrm{YMnO}_{3}$. (a) Fitting residuals $r_{w}$ of the high-symmetry NP and low-symmetry $\mathrm{P}$ and AP models to $I(Q)$, where $Q$ is the reciprocal space vector. The gray vertical line at $1223 \mathrm{~K}$ indicates the Curie temperature $T_{\mathrm{C}}$ above which the high-symmetry nonpolar structure can be inferred from the Bragg reflections. The gray shaded area between $800 \mathrm{~K}$ and $880 \mathrm{~K}$ separates low- and intermediatetemperature regions. (b) Lattice parameters $a$ and $c$; (c) $\mathrm{Y}$ corrugation $\Delta \mathrm{Y}$, apical tilt angle $\alpha_{\mathrm{A}}$, and planar tilt angle $\alpha_{\mathrm{P}}$; and (d) isotropic atomic displacement parameters $U$-all obtained from fitting the $\mathrm{P}$ ground-state model below $T_{\mathrm{C}}$ and the high-symmetry NP structure above $T_{\mathrm{C}}$ to the reciprocal space neutron time-of-flight data. (Representative Rietveld fits with the polar, antipolar, and nonpolar models are shown in Figs. S3-S5 of the Supplemental Material [26]).

Quantitative analysis of the average structure of $h-\mathrm{YMnO}_{3}$ was carried out by Rietveld refinements of the measured diffraction patterns with the three special space group models NP, P, and AP, for which representative fits are given in Figs. S3-S5 of the Supplemental Material [26]. In Fig. 2(a), we have presented the fit residuals $r_{w}$ for the $\mathrm{P}$ and AP models, found in the brim of the Mexican-hat energy landscape, below $T_{\mathrm{C}}$, and for the NP model, found at the top of the Mexican hat, above $T_{\mathrm{C}}$. Refinement parameters from fitting with the $\mathrm{P}$ model are presented in Figs. 2(b)-2(d). The models are described and visualized in Figs. 1(e) and $1(\mathrm{~g})$.

The models give adequate fits to the data with weighted profile agreement factors $\left(r_{w}\right)$ below $10 \%$. The polar model gives better agreement than the antipolar model for all temperatures below $T_{\mathrm{C}}$ [Fig. 2(a)]. Despite having fewer variables, the NP model gives a comparable $r_{w}$ to that of the
$\mathrm{P}$ model above $T_{\mathrm{C}}$. The most surprising observation is a discontinuous jump in $r_{w}$ extracted from the $\mathrm{P}$ and $\mathrm{AP}$ models at $800 \mathrm{~K}$. The lattice parameters [Fig. 2(b)] vary smoothly with temperature, also through $T_{\mathrm{C}}$. Above $T_{\mathrm{C}}$, the lattice parameter $a$ of the nonpolar model is multiplied by $\sqrt{3}$ for direct comparison with the tripled unit cell of the polar model.

There is no discontinuity of the lattice parameters at $800 \mathrm{~K}$ that might explain the change in $r_{w}$ there. However, there is a smooth drop-off from linear temperature dependence of the $c$-axis parameter with an onset at around $800 \mathrm{~K}$. This result is highlighted in the figure by the gray dashed line extrapolated from the low-temperature linear behavior. Such a drop-off has been noted previously [12], as has the extended region of zero thermal expansion of the $c$ axis above $T_{\mathrm{C}}$.

We now turn to the three order-parameter observables $\alpha_{\mathrm{A}}, \alpha_{\mathrm{P}}$, and $\Delta \mathrm{Y}$, whose temperature dependencies are shown in Fig. 2(c). All of these parameters have, by definition, a value of 0 in the high-symmetry nonpolar structure and are thus expected to decrease smoothly to zero on heating to $T_{\mathrm{C}}$, as they are all direct observables of the order-parameter amplitude $\mathcal{Q}$ (Fig. 1). The refinements of the $\alpha$ parameters to the polar model smoothly decrease with increasing temperature, becoming close to zero at $T_{\mathrm{C}}$, consistent with a continuous transformation. On the other hand, the corrugation parameter $\Delta \mathrm{Y}$ keeps a large value right up to $T_{\mathrm{C}}$, which would be expected for a discontinuous transition. From about $800-880 \mathrm{~K}$, the fits become unstable and the $\alpha$ parameters start to decrease faster than linearly, in agreement with previous studies [12], possibly explaining the jump in $r_{w}$ in panel (a).

The vanishing polyhedral tilting and persistent $\Delta Y$ could indicate a scenario in which the structure has off-centered $\mathrm{Y}$ ions combined with untilted $\mathrm{Mn}-\mathrm{O}_{5}$ polyhedra, which would give very long out-of-plane Y-O distances. We thus performed density functional calculations to check if this scenario is plausible and found that it is highly unfavorable (see Fig. S6 in the Supplemental Material [26]). We note that the $\mathrm{Y}$ and $\mathrm{O}$ isotropic atomic displacement parameters (ADPs) $U$ shown in Fig. 2(d) are anomalously large compared to the $\mathrm{Mn} U$, which is nonlinear upon approaching $T_{\mathrm{C}}$. There is also a discontinuous jump in the ADPs on moving into the high-symmetry nonpolar structure, suggesting that broken local symmetry persists above $T_{\mathrm{C}}$ [31-34]. Hence, we conclude that the structural behavior obtained from the reciprocal space refinements is physically unfeasible at the local scale, motivating the use of a local structure-sensitive method.

\section{B. Local structure}

In order to investigate the local structure, we perform a PDF analysis on the same neutron scattering data as analyzed in the previous section. Representative PDFs at different temperatures are shown in Fig. 3(a), plotted over a 


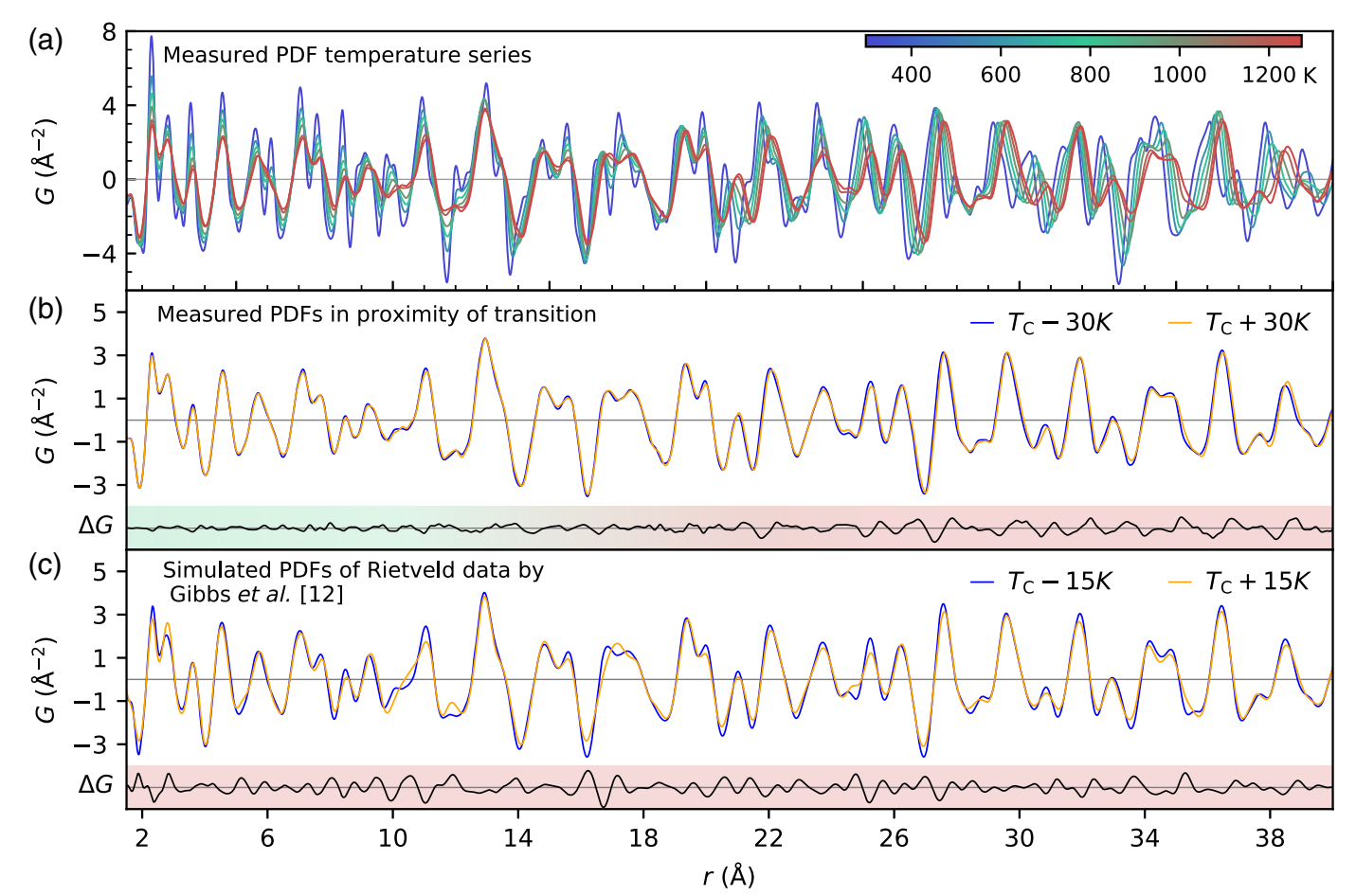

FIG. 3. (a) Experimental PDFs for 473-1273 K temperature interval in $100 \mathrm{~K}$ increments. The full set of PDF data on a denser $(\Delta \mathrm{T}=20 \mathrm{~K})$ grid and further details are provided in Figs. S7 and S8 of the Supplemental Material [26]. The data temperature is color coded, as indicated by the horizontal color bar. Comparison of PDFs for temperatures bracing the structural transition temperature $T_{\mathrm{C}}$ are shown in panels (b) and (c) as follows. Experimental PDFs from our measurements are displayed in panel (b). Simulated PDFs, calculated using the average structure information from Rietveld refined structures reported by Gibbs et al. [12] across the transition, are shown in panel (c). Corresponding difference curves are shown underneath. The shaded green and red areas behind the difference curve indicate, respectively, regions of low and high difference. The presence of length scales exhibiting different behavior can be readily evidenced by comparing the difference curves in panels (b) and (c). At low $r$, the measured PDFs change appreciably less than what would be expected if the average crystallographic picture were applicable to the local structure, as portrayed by the simulated PDFs. In contrast, the changes across the transition at high $r$ are of similar amplitude in both panels. Note that the structural phase transition temperature in Ref. [12] is $1258 \mathrm{~K}, 35 \mathrm{~K}$ higher than in our measurements. Comparison of simulated PDFs based on structural parameters from the Rietveld refinements of Gibbs et al. to that of this work is provided in Fig. S9 of the Supplemental Material [26].

wide range of $r$ (see detailed plots in Figs. S7 and S8 in the Supplemental Material [26].

PDF analysis utilizes both Bragg and diffuse scattering information, revealing time-averaged snapshots of the atomic structure on multiple length scales. In contrast to a conventional crystallographic approach that seeks the highest possible symmetry model consistent with the Bragg data component only, the PDF analysis explores whether such symmetry is broken on a nanometer length scale and, if so, how. The peak positions correspond to interatomic distances, while the widths of the peaks indicate distributions of interatomic distances due to thermal motion and also lower symmetry.

A characteristic of neutron PDFs of materials containing manganese (and other negative neutron scattering length materials) is that peaks corresponding to $\mathrm{Mn}-$ non-Mn pairs are negative. This characteristic is most clearly seen for the Mn-O nearest-neighbor bond length at around $2 \AA$. This peak is a doublet, as is clearly seen in the $298 \mathrm{~K}$ data in Figs. 3(a) and 4(a). On heating, this doublet broadens into an unstructured peak by $1273 \mathrm{~K}$ [Figs. 3(a) and 4(c)]. The increased broadening of the PDF with increasing temperature is clearly evident in Figs. 3 and 4.

Apart from thermal broadening effects, several other qualitative changes can be observed in the experimental PDFs with increasing temperature, as shown in Fig. 3(a). While we observe qualitative changes in PDF features at all length scales, these seem to become much more pronounced in the high- $r$ region (above $20 \AA$ ), suggesting length-scale-dependent structural changes on heating. The apparent order-disorder behavior of the system across $T_{\mathrm{C}}$, inferred from unphysical behavior of structural parameters derived from the Rietveld analysis, can also be further explored by comparing measured and simulated PDFs corresponding to temperatures straddling $T_{\mathrm{C}}$. If the transition were displacive, the PDFs would show clear changes of similar magnitude over the entire $r$ range. We illustrate this in Fig. 3(c) by comparing simulated PDFs using the 


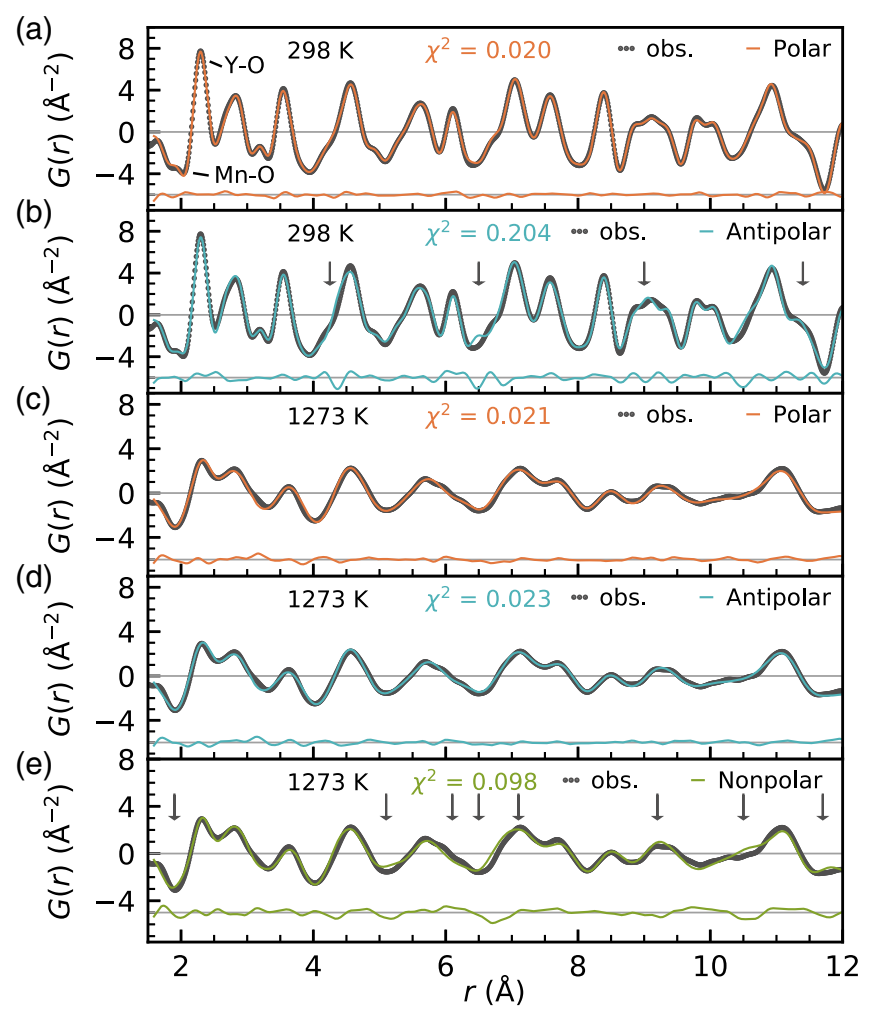

FIG. 4. Fits of the crystallographic models to the PDF data. The $G(r)$ at $298 \mathrm{~K}$ fitted over the range $r=1.6-12 \AA$ by the (a) lowsymmetry $\mathrm{P}$ and (b) AP models. Corresponding difference curves are shown beneath the PDFs, and the overall fit residuals $\chi^{2}$ are as indicated. Fits to the $1273 \mathrm{~K} G(r)$ data by the low-symmetry $\mathrm{P}$ and AP models over the same $r$ range are shown in panels (c) and (d), respectively. (e) Fit of the high-symmetry NP model to the 1273 K PDF data: Arrows mark regions of particularly bad fits. High fit residual and eventful difference curves demonstrate that the nonpolar model does not provide an adequate description of the local structure of the high-temperature phase. Fits of the PDF data for additional temperatures are shown in Figs. S10 and S11 of the Supplemental Material [26].

Rietveld refined structures reported by Gibbs et al. [12] that portray such a displacive picture. In this case, substantial structural changes are evident in the difference curve over the entire $r$ range considered [see Fig. 3(c)]. However, this is not what is observed when experimental PDFs measured at temperatures just below and just above $T_{\mathrm{C}}$ are compared, as can be seen in Fig. 3(b). It is evident that the observed structural changes are much smaller in the low- $r$ region up to about $15 \AA$, as compared to those in the $r$ range between 20 and $40 \AA$. This behavior is characteristic for an orderdisorder mechanism, where the local structure changes insignificantly across the transition, while the global symmetry change is evident in the measures of the average structure. The $r$ values of $16-20 \AA$ separating the local regime from the average regime correspond to approximately three unit cells in plane and 1.5-2 out of plane.

Quantitative information about the local structure can be further extracted by fitting the structural models to the PDF data. When symmetry breaking occurs only in the local structure but not in the average structure, it is customary to fit the PDF data with lower-than-crystallographic symmetry models over the relevant $r$ range [28]. We fitted the PDF data with models corresponding to the four space groups found in the Mexican-hat energy landscape: the P, AP, IM, and high-symmetry NP models over an $r$ range of $12 \AA$. This length scale corresponds to 1-2 unit cells and is well within the $r$ range implicated by the direct data comparisons, as discussed in relation to Fig. 3(b). Note that the IM model does not represent a single configuration of atoms, but it can describe any angle $\Phi$ of the order parameter in the brim of the Mexican hat, including the P and AP models.

In Fig. 4, we show representative PDFs at $298 \mathrm{~K}$, fitted with the polar and antipolar space groups, and at $1273 \mathrm{~K}$, fitted with the polar, antipolar, and nonpolar space groups. Importantly, and in agreement with other evidence of an order-disorder transition presented so far, the lowsymmetry polar model gives much better agreement with the $1273 \mathrm{~K}$ data set than the high-symmetry nonpolar model, as is apparent from the appreciably lower $\chi^{2}$ and clear appearance of misfit regions in the difference curve plotted below. While the antipolar structure fits the measured PDF significantly worse at room temperature [Fig. 4(b)], it provides a comparable fit to that of the polar model at $1273 \mathrm{~K}$. We will return to this observation later.

Next, we consider the PDF-derived lattice parameters and order parameter observables $\alpha_{\mathrm{A}}, \alpha_{\mathrm{P}}$, and $\Delta \mathrm{Y}$, shown in Fig. 5. The refined lattice parameters are in very good agreement with those obtained from Rietveld refinement, and they show the same behavior. The order-parameter observables are all expected to retain finite values above $T_{\mathrm{C}}$ for the now-established order-disorder transition. Indeed, we observe finite values of all three order-parameter observables. Surprisingly, however, they do not decrease by the same amount: $\alpha_{\mathrm{A}}$ decreases to about half its low- $T$ value, while $\alpha_{\mathrm{P}}$ retains about $80 \%$ of its low- $T$ value. Note that $\Delta \mathrm{Y}$ decreases to about $60 \%$ of its low- $T$ value. While these observations support the suggested order-disorder behavior, they also indicate that the P model used to fit the PDF data is not able to fully represent the structural changes upon heating.

This case is further substantiated by the uncertainties of $\Delta \mathrm{Y}$, which become significantly larger than those of $\alpha_{\mathrm{A}}$ and $\alpha_{\mathrm{P}}$ above $1000 \mathrm{~K}$, suggesting that $\mathrm{Y}$ displacements become progressively more difficult to determine within the polar model with increasing temperature. We note that, as with the average structure refinements, the atomic displacement parameters and/or their uncertainties experience an anomalous increase above about $800 \mathrm{~K}$, indicative of the increasing inadequacy of this model for describing the local structure as temperature increases.

Finally, we compare the quality of the fits with the $\mathrm{P}$ model to fits with the AP, IM, and high-symmetry NP models. The comparison of the fit residuals $r_{w}$ for the four 

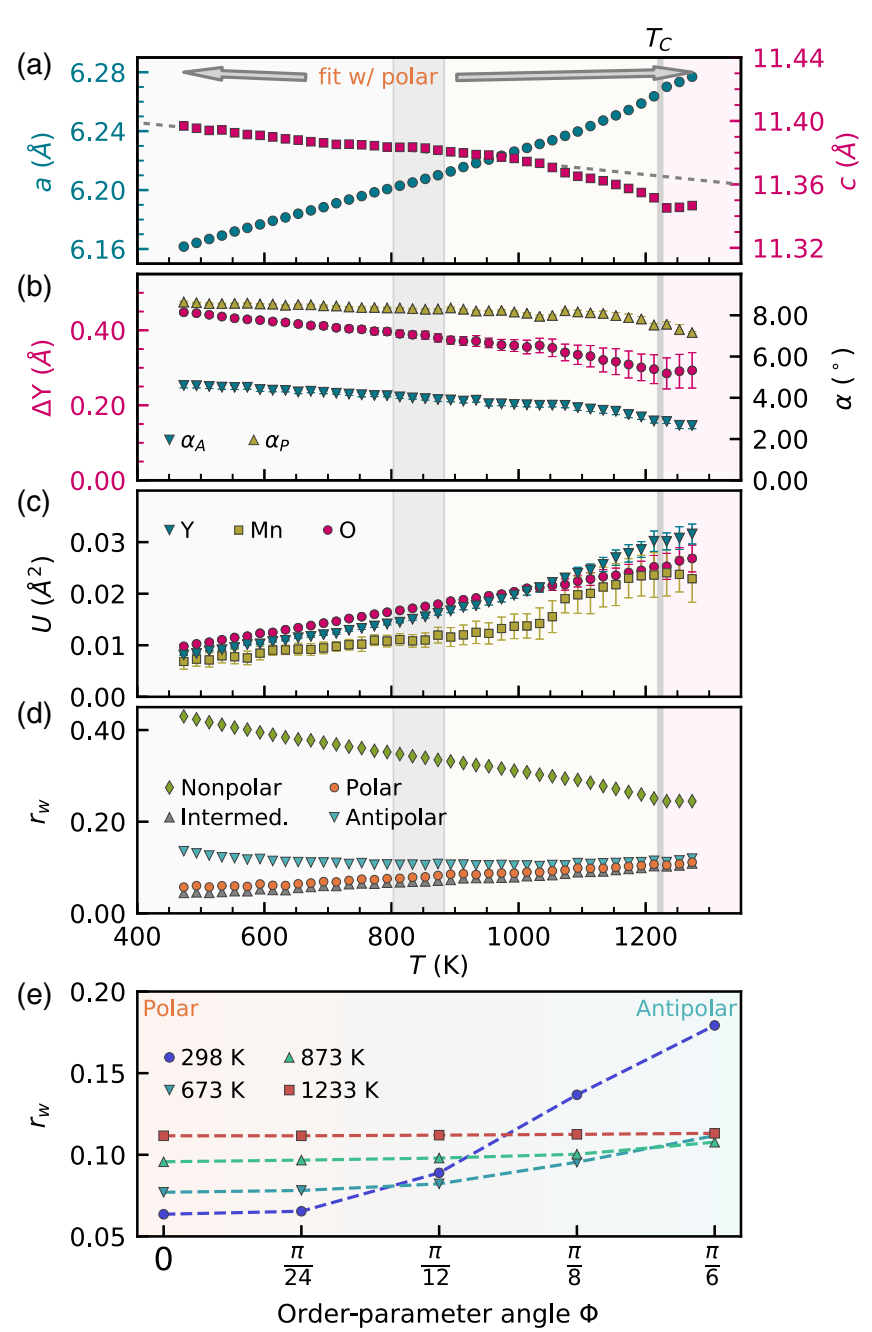

FIG. 5. Local structure refinements. (a) Refined lattice parameters from fitting $G(r)$ for $r=1.6-22 \AA$ with the polar space group model. (b) Y corrugation $\Delta \mathrm{Y}$, planar $\alpha_{\mathrm{P}}$, and apical $\alpha_{\mathrm{A}}$ bipyramidal tilt angles, and (c) atomic displacement parameters $U$ from fitting the polar model to the data in the range $r=1.6-12 \AA$ with fixed lattice parameters shown in panel (a) (see Supplemental Material [26] for details on the calculation of error bars). (d) Fit residual $r_{w}$ for fitting $G(r)$ between 1.6 and $12 \AA$ for the P model found at the minima and the AP model found at the local maxima in the brim of the Mexicanhat potential using fixed lattice parameters found from refinements between 1.6 and $22 \AA$. The grey vertical line at $1223 \mathrm{~K}$ shows the Curie temperature $T_{\mathrm{C}}$, and the grey shaded area between $800 \mathrm{~K}$ and $880 \mathrm{~K}$ shows the temperature interval where refinements become incoherent. (e) Fitting error $r_{w}$ as a function of the order-parameter angle for a rotation of the angle between the $\mathrm{P}$ and the AP structures at constant amplitude. The amplitude for each temperature is shown in panel (b). Comparisons of the refined order-parameter observables and ADPs of the polar model with those of the antipolar and intermediate space group models are shown in Figs. S12 and S13, respectively, of the Supplemental Material [26].

models [Fig. 5(d)] clearly shows that the AP model gives distinctly worse agreement than the $\mathrm{P}$ model at room temperature. As temperature increases, the AP model fits progressively improve, while the polar model fits deteriorate. This observation of stagnating or even increasing fitting error is anomalous, as for systems that do not exhibit structural changes the fit residuals are expected to decrease with heating as thermal fluctuations broaden the data (see, for example, the behavior of bulk FCC Ni in Fig. S14 of the Supplemental Material [26]). At around $1100 \mathrm{~K}$, the two models provide a similar, but not ideal, fit quality, suggesting that neither one of the two provides a complete description of the underlying local structure. Notably, anomalous jumps in $U$ are not fully reconciled by either the P, AP, or IM model of the local structure despite reasonably low fit residuals, indicating that the underlying local structure is of greater complexity than that described by these low-symmetry models (see Supplemental Material, Fig. S13). We observe that the IM model is comparable to the P model over the entire temperature range, with a slightly lower $r_{w}$, which we attribute to more degrees of freedom (16 for IM, 11 for P). This observation suggests that there is no other minimum at a different order-parameter angle $\Phi$ since the degrees of freedom in the intermediate model cover all angles in the brim of the Mexican hat.

We further examine the observation that the antipolar and polar models seem to fit equally well at higher temperatures by fitting the data to constructed models in which we manually rotate the order-parameter angle $\Phi$. The models are constructed starting from the refined structure for the $\mathrm{P}$ model and rotating $\Phi$ while keeping the order-parameter amplitude $\mathcal{Q}$ constant. This method is justified as the $\mathcal{Q}$ for refinements with all three subgroup models obtains comparable values (see Fig. S11 of the Supplemental Material [26]). We then fit the models at different temperatures, allowing the polar degrees of freedom as well as the ADPs to refined as presented in Fig. 5(e). As is evident from the figure, the roomtemperature data are much better fitted by the polar than the antipolar model, while as the temperature rises, the fit quality becomes independent of the order-parameter angle and the absolute fitting error increases overall. We note here that the invariance of the fitting error with respect to the order-parameter angle cannot be explained by the order-parameter amplitude $\mathcal{Q}$ going to zero, as the constructed models used here have the same amplitude of structural distortions as shown in panel (b), with the amplitude never falling below $50 \%$.

Thus, from our inspection of the fitting errors, we make four key observations on heating: (1) for the P model, $r_{w}$ increases; (2) for the P and AP models, $r_{w}$ becomes similar; (3) for the NP model, $r_{w}$, while decreasing, remains much larger; and (4) the fitting error becomes independent of the order-parameter angle when approaching $T_{\mathrm{C}}$.

These observations can be explained by a scenario in which the local structure in the high-symmetry phase is neither described by the NP model nor does it take any well-defined average low-symmetry structure. Instead, 
multiple different local configurations in the brim of the hat become represented simultaneously upon heating. Such a scenario is achieved by increasingly large fluctuations of $\Phi$, which increase the sampled phase space and thereby reduce the local symmetry. This kind of continuous disorder is the most likely explanation for the observed anomalies in the fits.

\section{Effective Hamiltonian analysis}

To better understand the unusual behavior indicated by our local structural analysis, we next analyze an effective Hamiltonian that describes the local structure and energetics of $\mathrm{YMnO}_{3}$. We focus specifically on whether our PDF analysis can be rationalized in the context of a competition between displacive and order-disorder behaviors. In the case of a conventional single-component order-parameter system described by a double-well potential, the two energy scales that determine whether displacive or order-disorder behavior dominates are (i) the energy cost per site, $V$, of removing the distortion that deforms the structure from the maximum to the minimum of the double well (this is the energy difference per unit cell between the high- and low-symmetry structures) and (ii) the intersite energy cost $W$ associated with changes in the distortion from one unit cell to the next (this is the additional energy of forming one locally undistorted unit cell in a background that is homogeneously distorted to the double-well minimum [35]). The displacive limit is reached when $V \ll W$ since the energy cost for removing the distortion of the units is less than the cost of introducing disorder between locally distorted units. In contrast, the order-disorder limit occurs when $W \ll V$, where locally ordered units are strongly favored and the cost of orienting them in different directions relative to each other is small [35-38]. The situation is more complex for $\mathrm{YMnO}_{3}$ with its two-component order parameter, and we consider the following effective Hamiltonian, which is a lattice version of the well-established Landau free energy of Refs. [20,21] (details in Appendix C). The resulting effective Hamiltonian is analogous to an $X Y$ model with variable spin sizes. We separate the Hamiltonian into the on-site Mexican-hat-potential contribution $\mathcal{H}_{V}$ and the intersite interaction energy contribution $\mathcal{H}_{W}$, which describes the energy cost of distorting the tilt pattern:

$$
\mathcal{H}=\mathcal{H}_{V}+\mathcal{H}_{W}
$$

where

$$
\begin{aligned}
& \mathcal{H}_{V}=\sum_{i} \frac{a}{2} Q^{2}\left(x_{i}\right)+\frac{b}{4} Q^{4}\left(x_{i}\right)+\frac{Q^{6}\left(x_{i}\right)}{6}\left(c+c^{\prime} \cos \left[6 \Phi\left(x_{i}\right)\right]\right)-g P\left(x_{i}\right) Q\left(x_{i}\right)^{3} \cos 3 \Phi\left(x_{i}\right)+\frac{g^{\prime}}{2} Q\left(x_{i}\right)^{2} P\left(x_{i}\right)^{2}+\frac{a_{p}}{2} P\left(x_{i}\right)^{2}, \\
& \mathcal{H}_{W}=\frac{J}{4} \sum_{i \neq j}\left[Q\left(x_{i}\right)-Q\left(x_{j}\right)\right]^{2}+2 Q\left(x_{i}\right)^{2}\left(1-\cos \left[\Phi\left(x_{i}\right)-\Phi\left(x_{j}\right)\right]\right) .
\end{aligned}
$$

Here, $x_{i}$ is the coordinate of the $i$ th lattice site, where each lattice site corresponds to a 30-atom unit cell, which is the smallest unit necessary to contain the trimerization distortions. The two-component order parameter $(\mathcal{Q}, \Phi)$ is a generalized coordinate representing the local trimerization amplitude $\mathcal{Q}\left(x_{i}\right)$ and angle $\Phi\left(x_{i}\right)$. The polarization $P\left(x_{i}\right)$ is improperly coupled to $\mathcal{Q}\left(x_{i}\right)$ and is the main cause of the energy barriers in the brim of the Mexican-hat potential. The parameters in $\mathcal{H}_{V}$ are taken directly from Ref. [21], and $J$ is extracted from the parametrized gradient terms in the same work (see Appendix C for details and a table of values). Our goal is to determine whether this Hamiltonian, with the parameters appropriate for $\mathrm{YMnO}_{3}$, describes a system in an order-disorder or displacive limit.

We begin by analyzing whether the local distortion amplitudes $\mathcal{Q}\left(x_{i}\right)$ remain finite or tend to zero at $T_{\mathrm{C}}$. We derive the following expressions for the cost of undistorting a site, $V_{\mathcal{Q}}$, as well as the interaction energy associated with undistorting a site within a homogeneously trimerized background, $W_{\mathcal{Q}}$,

$$
\begin{aligned}
V_{\mathcal{Q}} & =\min _{Q, \Phi, P}\left[\mathcal{H}_{V} / N\right] \approx 0.61 \mathrm{eV} \\
W_{\mathcal{Q}} & =\frac{J}{2} n \mathcal{Q}_{0}^{2} \approx 0.36 \mathrm{eV},
\end{aligned}
$$

using the parameters from Appendix C. Here, $n$ is the number of nearest neighbors, $N$ is the number of lattice sites, and $\mathcal{Q}_{0}=0.96 \AA$ is the $\mathcal{Q}$ value that minimizes the local energy (it finds the bottom of the Mexican hat). We find that $V_{\mathcal{Q}} / W_{\mathcal{Q}} \approx 1.7$. As explained above, the limit of a fully displacive system is given by $V_{\mathcal{Q}} / W_{\mathcal{Q}} \ll 1$, with the small $V_{\mathcal{Q}}$ energy cost favoring the local units undistorting when approaching $T_{\mathrm{C}}$, whereas in the opposite orderdisorder limit, $V_{\mathcal{Q}} / W_{\mathcal{Q}} \gg 1$, the local amplitude of the order parameter is preserved on approaching $T_{\mathrm{C}}$. Our value of $V_{\mathcal{Q}} / W_{\mathcal{Q}} \approx 1.7$ therefore points towards an orderdisorder-like behavior of the order-parameter amplitude. 
Given this finding that the local order-parameter amplitude remains finite at $T_{\mathrm{C}}$, we next explore the displacive versus order-disorder behavior of the order-parameter angle, corresponding to changes of the angle around the brim of the Mexican hat. In this case, we compare the on-site energy barrier between two local minima within the brim of the hat, $V_{\Phi}$, and the intersite energy cost $W_{\Phi}$ arising from a change of the trimerization angle by $2 \pi / 6$ (between two local minima). We obtain the values

$$
\begin{aligned}
V_{\Phi} & =\min _{Q, \Phi=0, P}\left[\mathcal{H}_{V} / N\right]-\min _{Q, \Phi=\frac{\pi}{6}, P}\left[\mathcal{H}_{V} / N\right] \approx 0.15 \mathrm{eV}, \\
W_{\Phi} & =J n \mathcal{Q}_{0}^{2}\left(1-\cos \frac{2 \pi}{6}\right) \approx 0.36 \mathrm{eV},
\end{aligned}
$$

giving $V_{\Phi} / W_{\Phi} \approx 0.4$. The small energy barriers between neighboring states in the brim of the hat allow the system to readily access a continuum of angles, leading to a predominantly displacive-like behavior of the orderparameter angle.

In summary, our analysis of the effective lattice Hamiltonian suggests a mixed scenario in which the order-parameter amplitude shows order-disorder behavior, and the order-parameter angle shows displacive behavior at $T_{\mathrm{C}}$. This case is consistent with the model that we proposed above to explain our PDF analysis, in that it predicts that the local order-parameter amplitude should not vanish [Figs. 5(b) and 5(d)] and that the P and AP models, which differ in their local order-parameter angle, should fit equally well at $T_{\mathrm{C}}$ [Fig. 5(d)] but still not fully represent the mix of IM phases present.

Finally, we performed a series of Monte Carlo (MC) simulations on the effective lattice Hamiltonian including only nearest-neighbor interactions. These simulations were performed using the Metropolis algorithm [39] on a $10 \times 10 \times 10$ unit cell with periodic boundary conditions, averaging six different runs over $\mathcal{O}\left(10^{6}\right)$ sweeps. To improve the convergence, we discretized the phase space in 36 equally spaced states in $[0,2 \pi]$ for $\Phi$ and 11 different states in the range $\left[0,1.25 \mathcal{Q}_{0}\right]$ for the amplitude $\mathcal{Q}\left(x_{i}\right)$. We then analyzed the behavior of the expectation value of the order parameter $\langle\mathcal{Q}\rangle$ and the local amplitude of the order parameter $\sqrt{\left\langle\mathcal{Q}^{2}\right\rangle}$ (see Appendix $\mathrm{C}$ for details). We found a $T_{\mathrm{C}}$ of about $2200 \mathrm{~K}$, which overestimates the experimental $T_{\mathrm{C}}$ by about $60 \%$. This finding is not unreasonable considering our use of the long-wavelength limit for calculating $J$ and our neglect of beyond-nearestneighbor and nonlocal phononic effects. The latter, in particular, will cause the depth of the local Mexican-hat potential to decrease with increasing temperature and will therefore decrease the transition temperature.

The observed behavior, presented in Fig. 6, is consistent with our analysis of the effective Hamiltonian above, as well as with our interpretation of the PDF measurements. When the system crosses $T_{\mathrm{C}}$, the local order-parameter
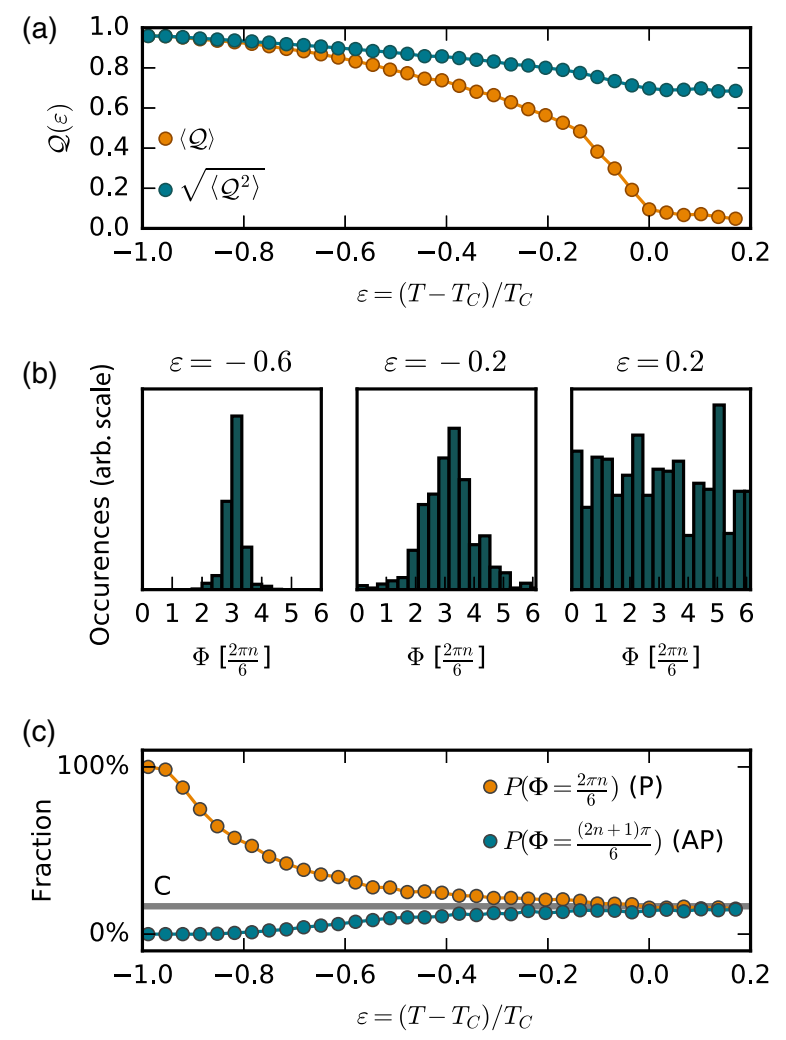

FIG. 6. Monte Carlo simulations of the effective Hamiltonian using the DFT parameters as described in the text. (a) Local orderparameter amplitude $\sqrt{\left\langle\mathcal{Q}^{2}\right\rangle}$ and its macroscopic expectation value $\langle\mathcal{Q}\rangle$, as a function of reduced temperature. Note that the local order parameter does not go to zero above $T_{\mathrm{C}}$, whereas its macroscopic expectation value vanishes above $T_{\mathrm{C}}$ due to the disordering of the order-parameter angle. (b) Histograms of the order parameter angle at a temperature well below $T_{\mathrm{C}}$ (left), just below $T_{\mathrm{C}}$ (center), and above $T_{\mathrm{C}}$ (right). We see that the angle is well defined at low temperatures, broadens upon heating, and can have any value above $T_{\mathrm{C}}$. (c) Probability $P$ that the states in the minima $(\Phi=[(2 \pi n) / 6])$ (brown circles) and the maxima $(\Phi=\{[(2 n+1) \pi] / 6\}$ ) (green circles) within the brim of the hat are occupied. At low $T$, only the minima are occupied $[P\{\Phi=[(2 \pi n) / 6]\}=100 \%]$, but at higher temperatures, the fractions converge towards the continuous limit marked by the line denoted "C," which corresponds to an equal distribution between all angles. The probabilities were extracted by creating 36 bins in $[0,2 \pi]$, and as a result, $\mathrm{C}$ has a $16.7 \%$ fraction.

amplitude $\sqrt{\left\langle\mathcal{Q}^{2}\right\rangle}$ is reduced by only around $20 \%$, while its macroscopic expectation value $\langle\mathcal{Q}\rangle$ goes to zero [Fig. 6(a)]. Considering the distribution of angles, at low temperatures the system is localized at one angle, while the distribution becomes broader around the expectation value upon heating, and disorders between all angles at $T>T_{\mathrm{C}}$ [Fig. 6(b)] [40]. We present a statistical analysis in Fig. 6(c), which shows that far away from $T_{\mathrm{C}}$ only the minimum energy states are occupied. The number of occupations of sites with intermediate order-parameter angles increases progressively with increasing temperature. The system ends up having an almost continuous 
distribution of order parameter angles over a substantial region below $T_{\mathrm{C}}$. Again, this result is consistent with the fits of the PDF data, with the AP and P phases having similar errors in a large region below $T_{\mathrm{C}}$ [Fig. 5(c)].

\section{DISCUSSION}

We have studied the structural evolution with temperature of hexagonal $\mathrm{YMnO}_{3}$ at both local and average length scales. The average structure from reciprocal-space Rietveld refinements is captured by the conventional $\mathrm{P}$ model below $T_{\mathrm{C}}$ and changes to the NP structure across the phase transition, in agreement with previous studies. However, on the local scale, both the symmetry and the structural evolution are very different from their average behavior, with the two components of the order parameter $(\mathcal{Q}, \Phi)$ even showing distinct behavior. Combining our experimental (total scattering + PDF) and theoretical $(\mathrm{DFT}+\mathrm{MC})$ results detailed above, we present the following conceptual order-disorder model for the structural phase transition (Fig. 7).

At low temperatures, the local structure corresponds to the P model of one of the six minima, consistent with the average structure [Fig. 7(a)] [41]. Upon heating above $800 \mathrm{~K}$, fluctuations of the angle $\Phi$ allow the local structure to access configurations of lower symmetry corresponding to the IM model, as illustrated Fig. 7(b). This region of the crossover corresponds to the reduced temperature starting at around $\epsilon=0.5$ in the MC simulations (Fig. 6), where $\left\langle\mathcal{Q}^{2}\right\rangle$ and $\langle\mathcal{Q}\rangle^{2}$ start to deviate from each other and where $P\{\Phi=(2 n+1) \pi / 6\}$ approaches $P\{\Phi=(2 n \pi) / 6\}$. This case explains the anomalous increase in fit residuals $r_{w}$ for the $\mathrm{P}$ model above $800 \mathrm{~K}$ and the increasingly similar $r_{w}$ for the different order-parameter angles [Fig. 5(e)]. This inadequacy of a single structural model is the reason for the artifacts observed in the Rietveld fits that we described in Sec. III A.

On approaching $T_{\mathrm{C}}$, these local fluctuations of $\Phi$ from its mean value become more pronounced. Close to, but below, $T_{\mathrm{C}}$, the measured local structure is a dynamic superposition of structural configurations that fluctuate within a continuum of values of $\Phi$ [Fig. 7(c)] while the average order parameter retains nonzero value. This scenario explains our $r_{w}$ fits (Fig. 5), where we saw a similar error of $\mathrm{P}$ and AP already in a region rather far below $T_{\mathrm{C}}$. It is consistent with our results from the effective Hamiltonian, where the distribution of order-parameter angles reveals an effectively flat free-energy landscape at high temperatures [Fig. 6(c)].

Above $T_{\mathrm{C}}$ [Fig. 7(d)], long-range order is lost and the system disorders between a continuum of angles $\Phi$, corresponding to all possible local IM structures with $P 3 c 1$ symmetry. Since the IM structures are polar, the structure remains polar above $T_{\mathrm{C}}$ on the local scale.

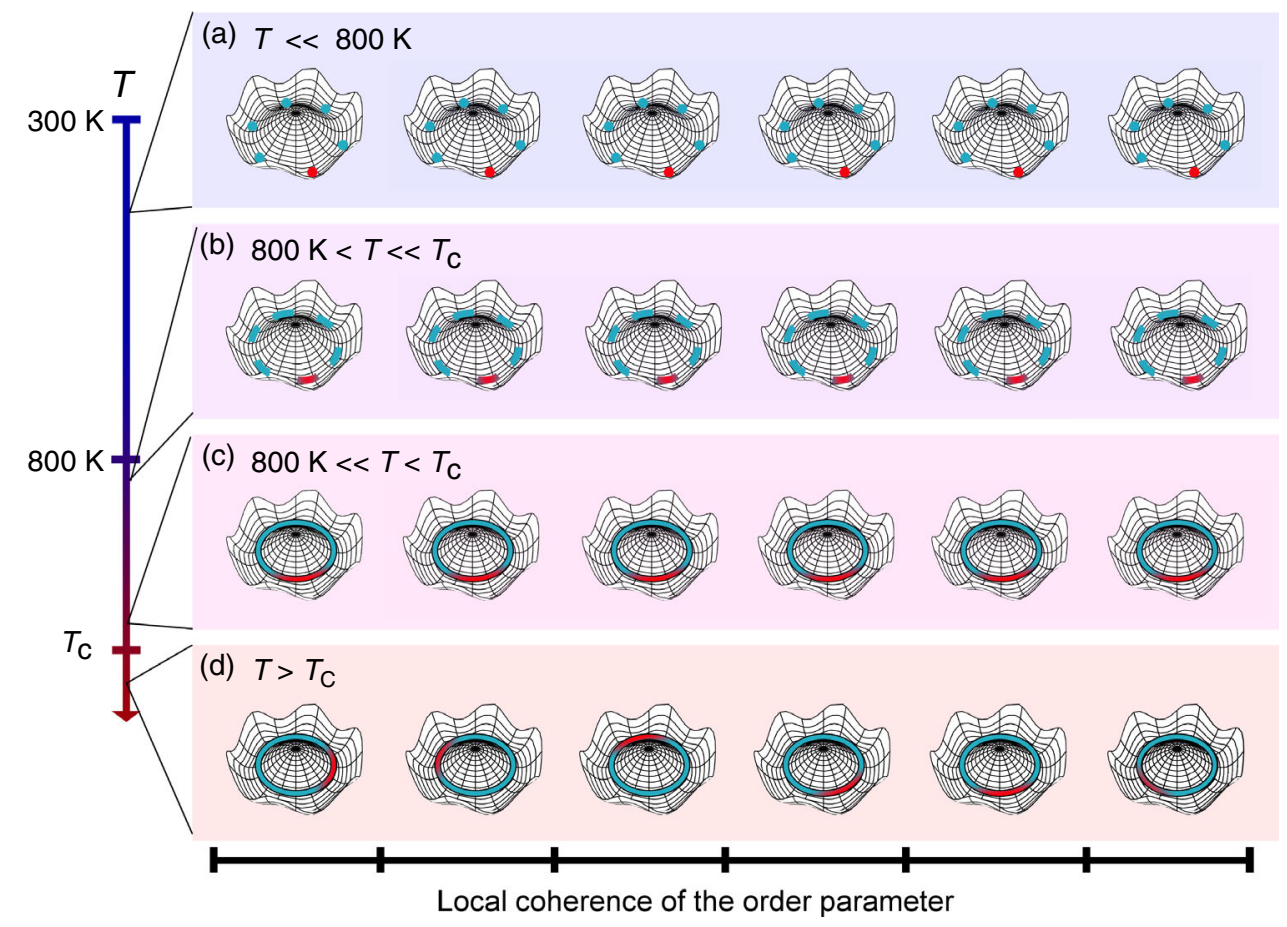

FIG. 7. Unconventional order-disorder transition of $\mathrm{YMnO}_{3}$. Local value of the order parameter in different temperature regions [panels (a)-(d)] within one domain. The blue lines indicate states accessible to the order parameter, and the red colored markings show the distribution of the order parameter. (a) In the low-temperature region, the order parameter is firmly fixed within one of the six ground states. (b) Between $800 \mathrm{~K}$ and $T_{\mathrm{C}}$, fluctuations smear out the local distribution of the order-parameter angle (red), but the average angle within each domain remains constant. (c) Close to $T_{\mathrm{C}}$, the energy landscape flattens. (d) At and above $T_{\mathrm{C}}$, coherence of the order parameter is lost and the system disorders between all possible states in the Mexican hat. 
This special order-disorder transition is unconventional in the sense that it does not disorder between the six degenerate ground states of the low-temperature ferroelectric P model but between a continuum of structures with all possible angles $\Phi$ of the order parameter.

This continuous disorder above $T_{\mathrm{C}}$ distinguishes the ferroelectric transition in $\mathrm{YMnO}_{3}$ from conventional orderdisorder transitions, e.g., $\mathrm{BaTiO}_{3}$ exhibiting disorder between different, but discrete, [111]-oriented ground states $[28,30]$. The continuous positional degree of freedom in $\mathrm{YMnO}_{3}$ is, in some ways, reminiscent of $\alpha-\mathrm{AlF}_{3}$ [33] and cristobalite [31], in which the structures are disordered with arbitrarily bent bonds between rigid polyhedral units. Here, however, because of the complexity of the structure, the disorder involves many atoms and bonds on a much larger length scale.

The high $T_{\mathrm{C}}$ of $\mathrm{YMnO}_{3}$ could also be important for the observed disorder. Notably, the corresponding $\mathrm{Pb}_{3} / \mathrm{mmc}^{-}$ $\mathrm{P}_{3} \mathrm{~cm}$ improper ferroelectric transition in $2 \mathrm{H}-\mathrm{BaMnO}_{3}$ [42] at $130 \mathrm{~K}$ is reported to be predominantly displacive [43]. However, the crystal structure of $2 \mathrm{H}-\mathrm{BaMnO}_{3}$ is fundamentally different in terms of polyhedral units and connectivity from that of $\mathrm{YMnO}_{3}$, making a direct comparison difficult. We hope that our work will motivate a detailed PDF study of the phase transition in $2 \mathrm{H}-\mathrm{BaMnO}_{3}$.

It is well established that there is only one phase transition in the hexagonal manganites at $T_{\mathrm{C}}$ with the polarization slowly emerging as a secondary effect [20-24], despite the many reported anomalies observed between $800 \mathrm{~K}$ and $T_{\mathrm{C}}$. Until now, these anomalies have not been fully explained. The model we have presented here, describing how the structural order parameter behaves in the local structure, provides an explanation for these anomalies. Entering the regime where fluctuations of the order parameter become increasingly important around $800 \mathrm{~K}$, the temperature dependence of the macroscopic order parameter is renormalized when approaching $T_{\mathrm{C}}$ $[35,44]$. This result will affect related macroscopic properties such as thermal expansion and specific heat [15], and can explain the thermal evolution of the lattice parameters: The reported change of slope $300-400 \mathrm{~K}$ below $T_{\mathrm{C}}$ and the flattening of the $c$ parameter just above $T_{\mathrm{C}}[12,15-17,45]$ can be expected from the coupling of the order parameter to strain. The temperature range of these anomalies also coincides with the reported Ginzburg temperature for $\mathrm{YMnO}_{3}$ [13], which is a measure of how far below $T_{\mathrm{C}}$ one must be to describe the system by mean-field theory. The accessible phase space for local order-parameter fluctuations far below $T_{\mathrm{C}}$ becomes limited to a small region around the local minimum. This freeze-in of fluctuations is in compliance with the reported glassy behavior of the system [46].

The gradual loss of long-range structural coherence upon approaching $T_{\mathrm{C}}$, as illustrated in Fig. 7 , is not easily detectable by conventional diffraction probing the average structure. Observations of $\alpha_{\mathrm{P}}$ and $\alpha_{\mathrm{A}}$ decreasing continuously to zero at $T_{\mathrm{C}}$ while $\Delta \mathrm{Y}$ goes abruptly to zero are demonstrated both by our own Rietveld refinements and others $[12,47]$. While discontinuity in one orderparameter observable across $T_{\mathrm{C}}$ has been suggested as a signature of a weakly first-order transition [47], the continuous molar volume (lattice parameters) and very subtle changes in the PDFs across $T_{\mathrm{C}}$ do not support this interpretation.

Finally, we note that order-disorder and displacive transitions show different features in their dynamical properties. For a purely displacive transition, the frequency of the soft phonon mode goes to zero at $T_{\mathrm{C}}$. On the other hand, the soft-mode frequency always remains nonzero for a purely order-disorder transition. Instead, one observes the emergence of an additional anharmonic relaxational mode at $\omega=0$, called the central mode [48-51]. While Gupta et al. [52] did not observe softening of any phonon branches below $T_{\mathrm{C}}$, Bouyanfif et al. [19] and Bansal et al. [53] observed a partial softening upon heating towards $T_{\mathrm{C}}$. However, it is not possible to identify a central mode from their data. These results suggest a mixed displacive and order-disorder character of the phase transition, and our model presented in Fig. 7 reconciles and explains these unusual spectroscopic observations.

We anticipate that the Mexican energy landscape will lead to unusual dynamics for the central mode. The influence of the central mode on properties like dielectric susceptibility have been studied for proper order-disorder ferroelectrics $[54,55]$ but may be very different in the case of this improper ferroelectric with unconventional disorder. We hope our work motivates future studies in this direction.

\section{CONCLUSIONS}

We discovered unconventional behavior across the improper ferroelectric transition in the hexagonal manganites where the amplitude of the two-dimensional order parameter is order-disorder-like, but the order-parameter angle behaves displacively. This unusual behavior is indicated by our finding that the local structure across $T_{\mathrm{C}}$ differs strongly from the established average structure symmetry and that the local low-symmetry distortions are conserved in the high-symmetry phase, both evidence of an order-disorder mechanism. Second, we observe that the low-symmetry distortions at high temperature are not restricted to specific minima but span a continuum of structures found in the brim of the Mexican-hat-shaped energy landscape, effectively lowering the local symmetry from $\mathrm{Pb}_{3} \mathrm{~cm}$ to $\mathrm{P} 3 \mathrm{c} 1$. Our findings reconcile decades of ambiguous reports on hexagonal manganites by showing that the reported anomalies in macroscopic data are caused by the onset of a continuum of local structures.

The only requirement for the presented behavior is the condensation of a disordered multicomponent order parameter with a small barrier between the different orderparameter directions. While such a continuous nature of 
the order parameter on the local scale is commonly found in magnetic structures, it is highly unusual in crystalline solids where the directionality of bonds and coupling to lattice strain normally favor discrete minima in the potential energy landscape. To the best of our knowledge, this work is the first report of such fundamentally different behavior of two components of a multicomponent order parameterone component showing order-disorder behavior and the other displacive.

Although reports of continuous structural disorder in crystalline solids are rare, the underlying structural and energetic requirements are not. Competing interactions and two characteristic energy barriers of different magnitude could lead to a frustrated structure where all bonds cannot be optimized simultaneously and where fluctuations of one order-parameter component can easily be masked by thermal vibrations. In addition to the improper ferroelectric hexagonal manganite $\mathrm{YMnO}_{3}$ studied here, similar structural and energetic features can also be found in magnetoelectric multiferroics [56], piezoelectrics at morphotropic phase boundaries [57], and relaxor ferroelectrics [58].

Hidden disorder is thus anticipated in a wide range of material systems with multicomponent order parameters and characteristic energy barriers of different magnitudes. Specific examples of systems where the continuous structural disorder introduced here could be relevant are the lowtemperature tetragonal phase of superconducting cuprates [59], which have been shown to exhibit order-disorder characteristics [60], the pyrochlore $\mathrm{Cd}_{2} \mathrm{Re}_{2} \mathrm{O}_{7}$ where Goldstone-like modes and order-disorder features have been reported [61-63], the shallow energy landscape of the layered ferroelectric perovskite $\mathrm{PbSr}_{2} \mathrm{Ti}_{2} \mathrm{O}_{7}$ [64], and (111)-strained perovskite oxides like $\mathrm{LaAlO}_{3}$ and $\mathrm{SrMnO}_{3}$, where the curvature of the Mexican-hat-shaped potential energy surface can be tuned by strain through the choice of substrate $[65,66]$.

This work illustrates that the atomic-scale mechanisms and underlying physics of phase transitions in complex crystalline solids with multicomponent order parameters and competing interactions can easily be overlooked if the local structure is not adequately probed, e.g., by total scattering methods.

\section{ACKNOWLEDGMENTS}

Experimental work was co-led by the groups at Brookhaven National Laboratory (BNL) and the Norwegian University of Science and Technology (NTNU). Theoretical work was led by ETH. Work at BNL was supported by U.S. DOE, Office of Science, Office of Basic Energy Sciences (DOE-BES) under Contract No. DE-SC0012704. The NTNU group acknowledges financial support from the Research Council of Norway (Project No. 231430) and NTNU. The ETH group was supported by ETH Zürich and Advanced Grant No. 291151 from the European Research Council. This research used resources at the Spallation Neutron Source, a DOE Office of Science User Facility operated by the Oak Ridge National Laboratory. Computational resources were provided by the Euler cluster at ETH, Zürich. Matt Tucker and Marshall McDonnell are acknowledged for assisting with the data collection for the neutron measurements. Matt Tucker is acknowledged for constructing TOPAS input files for reciprocal-space Rietveld refinements. Jörg Neuefeind and Pavol Juhas are acknowledged for assisting with manual data reduction and quality testing. Peter M. Derlet is acknowledged for constructive feedback on the manuscript.

\section{APPENDIX A: EXPERIMENTAL DETAILS, DATA REFINEMENT, AND SIMULATION OF PDFS}

Bulk powder of $\mathrm{YMnO}_{3}$ was prepared by firing uniaxially pressed pellets of dried and mixed $\mathrm{Y}_{2} \mathrm{O}_{3}$ (>99.99\%, Aldrich) and $\mathrm{Mn}_{2} \mathrm{O}_{3}$ (>99\%, Aldrich) twice for $24 \mathrm{~h}$ at $1573 \mathrm{~K}$ in air with intermediate grinding.

Neutron total scattering was performed at the NanoscaleOrdered Materials Diffractometer (NOMAD) [67] at the Spallation Neutron Source at Oak Ridge National Laboratory. A powder sample with mass of about $1.5 \mathrm{~g}$ was sealed in a 6-mm-diameter vanadium container and measured in an ILL-type vacuum furnace. The NOMAD detectors were calibrated using scattering from diamond standard powder, and Si standard powder was used to obtain the instrument parameter file for Rietveld refinements. The data were collected at room temperature and between 473 and $1273 \mathrm{~K}$ in steps of $20 \mathrm{~K}$ for $60 \mathrm{~min}$ at each temperature. Measurements of two subsequent temperature cycles between $1113 \mathrm{~K}$ and $1373 \mathrm{~K}$ on the same sample were performed to investigate chemical expansion as a function of oxygen loss and were found to be insignificant (see Fig. S15 of the Supplemental Material [26]). The structure factor $S(Q)$ was obtained by normalizing the scattering intensity to the scattering from a solid vanadium rod, and the background was subtracted using an identical, empty vanadium can. Pair distribution functions (PDF) were obtained by Fourier transform of $S(Q)$ with $Q_{\min }=$ $0.5 \AA^{-1}$ and $Q_{\max }=22 \AA^{-1}$.

The average crystal structure over the whole temperature range was determined by Rietveld refinements with the space groups $P 6_{3} / \mathrm{mmc}$ and $P 6_{3} \mathrm{~cm}$ [68], using TOPAS Academic v.5 [69]. PDFs were fitted to the same space groups at different ranges of $r$ using PDFgui [70]. Isotropic atomic displacement factors $(U)$ were used [26], and more details on calculation of error bars for $\Delta \mathrm{Y}, \alpha_{\mathrm{P}}$, and $\alpha_{\mathrm{A}}$ can be found in the Supplemental Methods [26]. We note that error bars for the real space and reciprocal space refinements cannot be quantitatively compared. Details of the PDF method can be found elsewhere [32]. Simulated PDFs were calculated using the PDFgui software with lattice 
parameters, atomic coordinates, and atomic displacement parameters from the Rietveld refinements, both with our data and with the data from Gibbs et al. [12]; they were scaled such that direct visual comparison could be made. The parameter $\delta_{1}$ was set to the value obtained for the relevant space group in the PDF refinements. For the assessment of fitting errors, we used the following quantities as implemented in PDFgui [70]:

$$
\chi^{2}=\frac{1}{N-p} \sum_{i=1}^{N}\left[G_{\mathrm{obs}}\left(r_{i}\right)-G_{\mathrm{calc}}\left(r_{i}\right)\right]^{2},
$$

where $N$ is the number of data points, $p$ is the degrees of freedom in the fit, $G_{\text {calc }}$ and $G_{\text {obs }}$ are the calculated and measured PDFs, and

$$
r_{w}=\sqrt{\frac{\sum_{i=1}^{N}\left[G_{\mathrm{obs}}\left(r_{i}\right)-G_{\mathrm{calc}}\left(r_{i}\right)\right]^{2}}{\sum_{i=1}^{N} G_{\mathrm{obs}}^{2}\left(r_{i}\right)}} .
$$

\section{APPENDIX B: COMPUTATIONAL DETAILS}

For our density functional calculations, we used the LDA $+U$ approximation [71,72] as implemented in the abinit PAW plane-wave code [73-76], with a cutoff energy of 30 Hartree and a $k$-point mesh of $6 \times 6 \times 2$. A collinear A-type magnetic ordering with a $U$ of $8 \mathrm{eV}$ on the $\mathrm{Mn} d$ orbitals was applied. While this magnetic ordering underestimates the band gap, it does not break the space group symmetry, which is important for this study. Here, 30-atom unit cells with lattice parameters from our PDF refinements were relaxed with respect to atomic positions such that the forces were converged within 1E-6 Ha/Bohr (see Supplemental Methods [26]).

\section{APPENDIX C: EFFECTIVE HAMILTONIAN}

The conventional expression for the Landau free energy is based on a continuous field model and gives the evolution of the average of the order parameter anywhere in the system. From first principles, we are only able to access properties at $0 \mathrm{~K}$.

In order to access information about the finitetemperature properties and order-parameter fluctuations, we map the Landau free energy into a discretized form.

TABLE I. Landau parameters from Ref. [21] and the derived nearest-neighbor coupling terms in this work.

\begin{tabular}{lcccc}
\hline \hline$a\left[\mathrm{eV} / \AA^{2}\right]$ & $b\left[\mathrm{eV} / \AA^{4}\right]$ & $c \mathrm{eV} /\left[\AA^{6}\right]$ & $c^{\prime} \mathrm{eV} /\left[\AA^{6}\right]$ & $g\left[\mathrm{eV} / \AA^{4}\right]$ \\
\hline-2.626 & 3.375 & 0.117 & 0.108 & 1.945 \\
\hline \hline$g^{\prime}\left[\mathrm{eV} / \AA^{4}\right]$ & $a_{p}\left[\mathrm{eV} / \AA^{2}\right]$ & $J_{z}\left[\mathrm{eV} / \AA^{2}\right]$ & $J_{x y}\left[\mathrm{eV} / \AA^{2}\right]$ & $\mathrm{J}\left[\mathrm{eV} / \AA^{2}\right]$ \\
\hline 9.931 & 0.866 & 0.120 & 0.140 & 0.133 \\
\hline \hline
\end{tabular}

We take the 30-atom unit cell of $\mathrm{YMnO}_{3}$ as the discretized unit since a local order parameter cannot be defined for a smaller unit. The terms in the Mexican-hat-potential contribution $\mathcal{H}_{V}$ are taken directly from the parameters calculated by Artyukhin et al. in Ref. [21] and reproduced in Table I. To extract the interaction energy terms $\mathcal{H}_{W}$, we mapped the gradient terms onto the $X Y$ model Hamiltonian,

$J \mathcal{Q}^{2} \cos \left[\Phi\left(x_{i}\right)-\Phi\left(x_{j}\right)\right]=J \mathcal{Q}^{2}\left(1-\frac{1}{2}\left(\Phi\left(x_{i}\right)-\Phi\left(x_{j}\right)\right)^{2}\right)$,

in order to enforce the necessary $2 \pi$ periodicity. Note that this expression reproduces the Landau free energy in the continuum limit.

We extract the coupling parameter $J$ by dividing each gradient term $\left(s_{Q}\right.$ in the notation of Ref. [21]) by the square of the relevant lattice constant $J_{i}=s_{i} / a_{i}^{2}$ (to approximate the energy cost of changing the trimerization between two unit cells) and then averaging the in-plane and out-of-plane values using $J=\left(J_{x y} J_{z}\right)^{1 / 3}$ [3].

We neglect any gradient contribution from the polar mode.

In our Monte Carlo simulations, we assume that the polarization is minimized on each lattice site, giving

$$
\frac{\partial \mathcal{H}}{\partial P\left(x_{i}\right)}=0
$$

leading to

$$
P\left(x_{i}\right)=\frac{g Q^{3}\left(x_{i}\right) \cos 3 \Phi}{g^{\prime} Q^{2}\left(x_{i}\right)+a_{p}}
$$

which we insert in Eq. (1).

To calculate the expectation values of the order parameter, we need to project it onto an arbitrary axis, for example,

$$
\left(\mathcal{Q}_{1}\left(x_{i}\right), \mathcal{Q}_{2}\left(x_{i}\right)\right)=\left(\mathcal{Q}\left(x_{i}\right) \cos \Phi\left(x_{i}\right), \mathcal{Q}\left(x_{i}\right) \sin \Phi\left(x_{i}\right)\right) .
$$

From this calculation, the macroscopic expectation value for the order parameter can be calculated using

$$
\langle\mathcal{Q}\rangle=\sqrt{\left\langle\mathcal{Q}_{1}\right\rangle^{2}+\left\langle\mathcal{Q}_{2}\right\rangle^{2}}
$$

and the expectation value of the local order-parameter amplitude is given by

$$
\sqrt{\left\langle\mathcal{Q}^{2}\right\rangle}=\sqrt{\left\langle\mathcal{Q}_{1}^{2}+\mathcal{Q}_{2}^{2}\right\rangle} .
$$


[1] S. M. Griffin, M. Lilienblum, K. T. Delaney, Y. Kumagai, M. Fiebig, and N. A. Spaldin, Scaling Behavior and Beyond Equilibrium in the Hexagonal Manganites, Phys. Rev. X 2 , 041022 (2012).

[2] S.-Z. Lin, X. Wang, Y. Kamiya, G.-W. Chern, F. Fan, D. Fan, B. Casas, Y. Liu, V. Kiryukhin, W. H. Zurek, C. D. Batista, and S.-W. Cheong, Topological Defects as Relics of Emergent Continuous Symmetry and Higgs Condensation of Disorder in Ferroelectrics, Nat. Phys. 10, 970 (2014).

[3] Q. N. Meier, M. Lilienblum, S. M. Griffin, K. Conder, E. Pomjakushina, Z. Yan, E. Bourret, D. Meier, F. Lichtenberg, E. K. H. Salje, N. A. Spaldin, M. Fiebig, and A. Cano, Global Formation of Topological Defects in the Multiferroic Hexagonal Manganites, Phys. Rev. X 7, 041014 (2017).

[4] N. Fujimura, T. Ishida, T. Yoshimura, and T. Ito, Epitaxially Grown $\mathrm{YMnO}_{3}$ Film: New Candidate for Nonvolatile Memory Devices, Appl. Phys. Lett. 69, 1011 (1996).

[5] Y. Kumagai, A. A. Belik, M. Lilienblum, N. Leo, M. Fiebig, and N. A. Spaldin, Observation of Persistent Centrosymmetricity in the Hexagonal Manganite Family, Phys. Rev. B 85, 174422 (2012).

[6] T. Lottermoser, M. Fiebig, and D. Fröhlich, Symmetry and Coupling of Magnetic and Electric Order Parameters in $\mathrm{YMnO}_{3}$, J. Appl. Phys. 91, 8251 (2002).

[7] T. Choi, Y. Horibe, H. T. Yi, Y. J. Choi, W. Wu, and S.-W. Cheong, Insulating Interlocked Ferroelectric and Structural Antiphase Domain Walls in Multiferroic $\mathrm{YMnO}_{3}$, Nat. Mater. 9, 253 (2010).

[8] T. Jungk, Á. Hoffmann, M. Fiebig, and E. Soergel, Electrostatic Topology of Ferroelectric Domains in $\mathrm{YMnO}_{3}, \mathrm{Appl}$. Phys. Lett. 97, 012904 (2010).

[9] D. Meier, J. Seidel, A. Cano, K. Delaney, Y. Kumagai, M. Mostovoy, N. A. Spaldin, R. Ramesh, and M. Fiebig, Anisotropic Conductance at Improper Ferroelectric Domain Walls, Nat. Mater. 11, 284 (2012).

[10] Y. Geng, N. Lee, Y. J. Choi, S.-W. Cheong, and W. Wu, Collective Magnetism at Multiferroic Vortex Domain Walls, Nano Lett. 12, 6055 (2012).

[11] J. A. Mundy, J. Schaab, Y. Kumagai, A. Cano, M. Stengel, I. P. Krug, D. M. Gottlob, H. Doganay, M. E. Holtz, R. Held, Z. Yan, E. Bourret, C. M. Schneider, D. G. Schlom, D. A. Muller, R. Ramesh, N. A. Spaldin, and D. Meier, Functional Electronic Inversion Layers at Ferroelectric Domain Walls, Nat. Mater. 16, 622 (2017).

[12] A.S. Gibbs, K.S. Knight, and P. Lightfoot, HighTemperature Phase Transitions of Hexagonal $\mathrm{YMnO}_{3}$, Phys. Rev. B 83, 094111 (2011).

[13] T. Katsufuji, M. Masaki, A. Machida, M. Moritomo, K. Kato, E. Nishibori, M. Takata, M. Sakata, K. Ohoyama, K. Kitazawa, and H. Takagi, Crystal Structure and Magnetic Properties of Hexagonal $R \mathrm{MnO}_{3}(R=Y, L u$, and $S c)$ and the Effect of Doping, Phys. Rev. B 66, 134434 (2002).

[14] J. Kim, K. C. Cho, Y. M. Koo, Kun P. Hong, and N. Shin, $Y-O$ Hybridization in the Ferroelectric Transition of $\mathrm{YMnO}_{3}$, Appl. Phys. Lett. 95, 132901 (2009).

[15] G. Nénert, M. Pollet, S. Marinel, G. R. Blake, A. Meetsma, and T. T. M. Palstra, Experimental Evidence for an Intermediate Phase in the Multiferroic $\mathrm{YMnO}_{3}$, J. Phys. Condens. Matter 19, 466212 (2007).
[16] T. A. Tyson, T. Wu, H. Y. Chen, J. Bai, K. H. Ahn, K. I. Pandya, S. B. Kim, and S.-W. Cheong, Measurements and Ab Initio Molecular Dynamics Simulations of the High Temperature Ferroelectric Transition in Hexagonal $\mathrm{RMnO}_{3}$, J. Appl. Phys. 110, 084116 (2011).

[17] S. M. Selbach, A. Nordli Løvik, K. Bergum, J. R. Tolchard, M.-A. Einarsrud, and T. Grande, Crystal Structure, Chemical Expansion and Phase Stability of $\mathrm{HoMnO}_{3}$ at High Temperature, J. Solid State Chem. 196, 528 (2012).

[18] I.-K. Jeong, N. Hur, and T. Proffen, High-Temperature Structural Evolution of Hexagonal Multiferroic $\mathrm{YMnO}_{3}$ and $\mathrm{YbMnO}_{3}$, J. Appl. Crystallogr. 40, 730 (2007).

[19] H. Bouyanfif, A. M. Salah, M. Zaghrioui, and M. El Marssi, High-Temperature Lattice-Dynamics Evolution of $\mathrm{YMnO}_{3}$ and $\mathrm{YbMnO}_{3}$, Phys. Rev. B 91, 224104 (2015).

[20] C. J. Fennie and K. M. Rabe, Ferroelectric Transition in $\mathrm{YMnO}_{3}$ from First Principles, Phys. Rev. B 72, 100103(R) (2005).

[21] S. Artyukhin, K. T. Delaney, N. A. Spaldin, and M. Mostovoy, Landau Theory of Topological Defects in Multiferroic Hexagonal Manganites, Nat. Mater. 13, 42 (2014).

[22] R. I. Thomson, T. Chatterji, C. J. Howard, T. T. M. Palstra, and M. A. Carpenter, Elastic Anomalies Associated with Structural and Magnetic Phase Transitions in Single Crystal Hexagonal $\mathrm{YMnO}_{3}$, J. Phys. Condens. Matter 26, 045901 (2014).

[23] A. Cano, Hidden Order in Hexagonal $R \mathrm{MnO}_{3}$ Multiferroics $(R=\mathrm{Dy}-\mathrm{Lu}$, In, $Y$, and Sc), Phys. Rev. B 89, 214107 (2014).

[24] M. Lilienblum, T. Lottermoser, S. Manz, S. M. Selbach, A. Cano, and M. Fiebig, Ferroelectricity in the Multiferroic Hexagonal Manganites, Nat. Phys. 11, 1070 (2015).

[25] B. B. Van Aken, T. T. M. Palstra, A. Filippetti, and N. A. Spaldin, The Origin of Ferroelectricity in Magnetoelectric $\mathrm{YMnO}_{3}$, Nat. Mater. 3, 164 (2004).

[26] See Supplemental Material at http://link.aps.org/ supplemental/10.1103/PhysRevX.9.031001 for more details on data collection and refinement, and computational results.

[27] M. Šafránková, J. Fousek, and S. A. Kižaev, Domains in Ferroelectric $\mathrm{YMnO}_{3}$, Czech. J. Phys. B 17, 559 (1967).

[28] G. H. Kwei, S. J. L. Billinge, S.-W. Cheong, and J. G. Saxton, Pair-Distribution Functions of Ferroelectric Perovskites: Direct Observation of Structural Ground States, Ferroelectrics 164, 57 (1995).

[29] D. A. Keen and A. L. Goodwin, The Crystallography of Correlated Disorder, Nature (London) 521, 303 (2015).

[30] M. S. Senn, D. A. Keen, T. C. A. Lucas, J. A. Hriljac, and A. L. Goodwin, Emergence of Long-Range Order in $\mathrm{BaTiO}_{3}$ from Local Symmetry-Breaking Distortions, Phys. Rev. Lett. 116, 207602 (2016).

[31] M. G. Tucker, M. P. Squires, M. T. Dove, and D. A. Keen, Dynamic Structural Disorder in Cristobalite: Neutron Total Scattering Measurement and Reverse Monte Carlo Modelling, J. Phys. Condens. Matter 13, 403 (2001).

[32] T. Egami and S.J.L. Billinge, Underneath the Bragg Peaks, Structural Analysis of Complex Materials (Elsevier, Oxford, 2003). 
[33] P. J. Chupas, S. Chaudhuri, J. C. Hanson, X. Qiu, P. L. Lee, S. D. Shastri, S. J. L. Billinge, and C. P. Grey, Probing Local and Long-Range Structure Simultaneously: An In Situ Study of the High-Temperature Phase Transition of $\alpha-\mathrm{AlF}_{3}$, J. Am. Chem. Soc. 126, 4756 (2004).

[34] X. Qiu, T. Proffen, J. F. Mitchell, and S. J. L. Billinge, Orbital Correlations in the Pseudocubic $O$ and Rhombohedral $R$ Phases of $\mathrm{LaMnO}_{3}$, Phys. Rev. Lett. 94, 177203 (2005).

[35] A. D. Bruce, Structural Phase Transitions. II. Static Critical Behaviour, Adv. Phys. 29, 111 (1980).

[36] E. K. H. Salje, Crystallography and Structural Phase Transitions, An Introduction, Acta Crystallogr. Sect. A 47, 453 (1991).

[37] S. Padlewski, A. K. Evans, C. Ayling, and V. Heine, Crossover between Displacive and Order/Disorder Behaviour in the $\mathrm{Phi}^{4}$ Model, J. Phys. Condens. Matter 4, 4895 (1992).

[38] M. T. Dove, Theory of Displacive Phase Transitions in Minerals, Am. Mineral. 82, 213 (1997).

[39] N. Metropolis, A. W. Rosenbluth, M. N. Rosenbluth, A. H. Teller, and E. Teller, Equation of State Calculations by Fast Computing Machines, J. Chem. Phys. 21, 1087 (1953), https://doi.org/10.1063/1.1699114.

[40] One can tune the Hamiltonian to become displacive when lowering the on-site energy by reducing $a$, and one can tune towards discrete disorder by lowering the intersite interaction $J$, as is expected according to our defined criteria above (we show MC on two examples in Supplemental Material, Fig. S16 [26]).

[41] We also checked for signs of higher-order terms in the free energy previously proposed [23] but found no evidence of such hidden order.

[42] J. Varignon and P. Ghosez, Improper Ferroelectricity and Multiferroism in 2H-BaMnO 3 , Phys. Rev. B 87, 140403(R) (2013).

[43] S. Kamba, D. Nuzhnyy, M. Savinov, P Tolédano, V. Laguta, P. Brázda, L. Palatinus, F. Kadlec, F. Borodavka, C. Kadlec, P. Bednyakov, V. Bovtun, M. Kempa, D. Kriegner, J. Drahokoupil, J. Kroupa, J. Prokleška, K. Chapagain, B. Dabrowski, and V. Goian, Unusual Ferroelectric and Magnetic Phases in Multiferroic $2 \mathrm{H}-\mathrm{BaMnO}_{3}$ Ceramics, Phys. Rev. B 95, 174103 (2017).

[44] T. Schneider and E. Stoll, Molecular-Dynamics Study of Structural-Phase Transitions. I. One-Component Displacement Models, Phys. Rev. B 13, 1216 (1976).

[45] H. D. Zhou, J.C. Denyszyn, and J. B. Goodenough, Effect of $\mathrm{Ga}$ Doping on the Multiferroic Properties of $\mathrm{RMn}_{1-x} \mathrm{Ga}_{x} \mathrm{O}_{3} \mathrm{R}=($ Ho, Y), Phys. Rev. B 72, 224401 (2005).

[46] A. Barbour, A. Alatas, Y. Liu, C. Zhu, B. M. Leu, X. Zhang, A. Sandy, M. S. Pierce, X. Wang, S.-W. Cheong, and H. You, Partial Glass Isosymmetry Transition in Multiferroic Hexagonal $\mathrm{ErMnO}_{3}$, Phys. Rev. B 93, 054113 (2016).

[47] H. Sim, J. Jeong, H. Kim, S.-W. Cheong, and J.-G. Park, Studies on the High-Temperature Ferroelectric Transition of Multiferroic Hexagonal Manganite $\mathrm{RMnO}_{3}$, J. Phys. Condens. Matter 30, 105601 (2018).

[48] S. M. Shapiro, J. D. Axe, G. Shirane, and T. Riste, Critical Neutron Scattering in $\mathrm{SrTiO}_{3}$ and $\mathrm{KMnF}_{3}$, Phys. Rev. B 6 , 4332 (1972).
[49] J. Petzelt, G. V. Kozlov, and A. A. Volkov, Dielectric Spectroscopy of Paraelectric Soft Modes, Ferroelectrics 73, 101 (1987).

[50] Y. Onodera, Dynamical Response of Ferroelectrics in Terms of a Classical Anharmonic-Oscillator Model, J. Phys. Soc. Jpn. 73, 1216 (2004).

[51] J. Hlinka, T. Ostapchuk, D. Nuzhnyy, J. Petzelt, P. Kuzel, C. Kadlec, P. Vanek, I. Ponomareva, and L. Bellaiche, Coexistence of the Phonon and Relaxation Soft Modes in the Terahertz Dielectric Response of Tetragonal $\mathrm{BaTiO}_{3}$, Phys. Rev. Lett. 101, 167402 (2008).

[52] M. K. Gupta, R. Mittal, M. Zbiri, N. Sharma, S. Rols, H. Schober, and S. L. Chaplot, Spin-Phonon Coupling and High-Temperature Phase Transition in Multiferroic Material $\mathrm{YMnO}_{3}$, J. Mater. Chem. C 3, 11717 (2015).

[53] D. Bansal, J. L. Niedziela, R. Sinclair, V. O. Garlea, D. L. Abernathy, S. Chi, Y. Ren, H. Zhou, and O. Delaire, Momentum-Resolved Observations of the Phonon Instability Driving Geometric Improper Ferroelectricity in Yttrium Manganite, Nat. Commun. 9, 818 (2018).

[54] Y. Girshberg and Y. Yacoby, Ferroelectric Phase Transitions in Perovskites with Off-Center Ion Displacements, Solid State Commun. 103, 425 (1997).

[55] J. Weerasinghe, L. Bellaiche, T. Ostapchuk, P. Kužel, C. Kadlec, S. Lisenkov, I. Ponomareva, and J. Hlinka, Emergence of Central Mode in the Paraelectric Phase of Ferroelectric Perovskites, MRS Commun. 3, 41 (2013).

[56] M. Fiebig, M. Lottermoser, D. Meier, and M. Trassin, The Evolution of Multiferroics, Nat. Mater. Rev. 1, 16046 (2016).

[57] M. Ahart, M. Somayazulu, R. Cohen, P. Ganesh, P. Dera, H.-K Mao, R. Hemley, Y. Ren, H.-P. Liermann, and Z. Wu, Origin of Morphotropic Phase Boundaries in Ferroelectrics, Nature (London) 451, 545 (2008).

[58] B. Noheda, D. E. Cox, G. Shirane, S.-E. Park, L. E. Cross, and Z. Zhong, Polarization Rotation via a Monoclinic Phase in the Piezoelectric 92\% $\mathrm{PbZn}_{1 / 3} \mathrm{Nb}_{2 / 3} \mathrm{O}_{3}-8 \%$ $\mathrm{PbTiO}_{3}$, Phys. Rev. Lett. 86, 3891 (2001).

[59] W. E. Pickett, R. E. Cohen, and H. Krakauer, Lattice Instabilities, Isotope Effect, and High- $T_{c}$ Superconductivity in $\mathrm{La}_{2-x} \mathrm{Ba}_{x} \mathrm{CuO}_{4}$, Phys. Rev. Lett. 67, 228 (1991).

[60] E. S. Bozin, R. Zhong, K. R. Knox, G. Gu, J. P. Hill, J. M. Tranquada, and S. J. L. Billinge, Reconciliation of Local and Long-Range Tilt Correlations in Underdoped $\mathrm{La}_{2-x} \mathrm{Ba}_{x} \mathrm{CuO}_{4} \quad(0=<x=<0.155)$, Phys. Rev. B 91, 054521 (2015).

[61] C. S. Knee, J. Holmlund, J. Andreasson, M. Käll, S. G. Eriksson, and L. Börjesson, Order-Disorder-Order Phase Transitions in the Pyrochlore Superconductor $\mathrm{Cd}_{2} \mathrm{Re}_{2} \mathrm{O}_{7}$, Phys. Rev. B 71, 214518 (2005).

[62] C. A. Kendziora, I. A. Sergienko, R. Jin, J. He, V. Keppens, B. C. Sales, and D. Mandrus, Goldstone-Mode Phonon Dynamics in the Pyrochlore $\mathrm{Cd}_{2} \mathrm{Re}_{2} \mathrm{O}_{7}$, Phys. Rev. Lett. 95, 125503 (2005).

[63] J. C. Petersen, M. D. Caswell, J. S. Dodge, I. A. Sergienko, J. He, R. Jin, and D. Mandrus, Nonlinear Optical Signatures of the Tensor Order in $\mathrm{Cd}_{2} \mathrm{Re}_{2} \mathrm{O}_{7}$, Nat. Phys. 2, 605 (2006).

[64] S. M. Nakhmanson and I. Naumov, Goldstone-like States in a Layered Perovskite with Frustrated Polarization: A 
First-Principles Investigation of $\mathrm{PbSr}_{2} \mathrm{Ti}_{2} \mathrm{O}_{7}$, Phys. Rev. Lett. 104, 097601 (2010).

[65] A. Marthinsen, S. M. Griffin, M. Moreau, T. Grande, T. Tybell, and S. M. Selbach, Goldstone-like Phonon Modes in a (111)-Strained Perovskite, Phys. Rev. Mater. 2, 014404 (2018).

[66] M. Moreau, A. Marthinsen, S. M. Selbach, and T. Tybell, First-Principles Study of the Effect of (111) Strain on Octahedral Rotations and Structural Phases of $\mathrm{LaAlO}_{3}$, Phys. Rev. B 95, 064109 (2017).

[67] J. Neuefeind, M. Feygenson, J. Carruth, R. Hoffmann, and K. K. Chipley, The Nanoscale Ordered Materials Diffractometer NOMAD at the Spallation Neutron Source SNS, Nucl. Instrum. Meth. Phys., Res. B 287, 68 (2012).

[68] B. B. van Aken, A. Meetsma, and T. T. M. Palstra, Hexagonal $\mathrm{YMnO}_{3}$, Acta Crystallogr. Sect. C 57, 230 (2001).

[69] J. P. Perdew and Y. Wang, Accurate and Simple Analytic Representation of the Electron-Gas Correlation Energy, Phys. Rev. B 45, 13244 (1992).

[70] C. L. Farrow, P. Juhas, J. W. Liu, D. Bryndin, E. S. Bozin, J. Bloch, Th. Proffen, and S. J.L. Billinge, PDFfit2 and PDFgui: Computer Programs for Studying Nanostructure in Crystals, J. Phys. Condens. Matter 19, 335219 (2007).
[71] J. P. Perdew and Y. Wang, Accurate and Simple Analytic Representation of the Electron-Gas Correlation Energy, Phys. Rev. B 45, 13244 (1992).

[72] A. I. Liechtenstein, V. I. Anisimov, and J. Zaanen, Density-Functional Theory and Strong Interactions: Orbital Ordering in Mott-Hubbard Insulators, Phys. Rev. B 52, R5467 (1995).

[73] X. Gonze, J. M. Beuken, R. Caracas, F. Detraux, M. Fuchs, G. M. Rignanese, L. Sindic, M. Verstraete, G. Zerah, F. Jollet, M. Torrent, A. Roy, M. Mikami, Ph. Ghosez, J. Y. Raty, and D.C. Allan, First-Principles Computation of Material Properties: The ABINIT Software Project, Comput. Mater. Sci. 25, 478 (2002).

[74] X. Gonze, A Brief Introduction to the ABINIT Software Package, Z. Kristallog. Cryst. Mater. 220, 558 (2005).

[75] M. Torrent, F. Jollet, F. Bottin, G. Zérah, and X. Gonze, Implementation of the Projector Augmented-Wave Method in the ABINIT Code: Application to the Study of Iron under Pressure, Comput. Mater. Sci. 42, 337 (2008).

[76] B. Amadon, F. Jollet, and M. Torrent, $\gamma$ and $\beta$ Cerium: LDA $+U$ Calculations of Ground-State Parameters, Phys. Rev. B 77, 155104 (2008). 